\title{
COMPORTAMENTO RACIONAL E DESEMPENHO DA ANÁLISE GRÁFICA NO MERCADO ACIONÁRIO BRASILEIRO
}

\author{
THE PERFORMANCE OF RATIONAL CHOICE AND TECHNICAL \\ ANALYSIS IN BRAZILIAN STOCK MARKET
}

Recebido - 24.01.2011

Aceito - 28.04.2011

José de Pietro Neto ${ }^{1}$ e Otavio Ribeiro de Medeiros ${ }^{2}$

\begin{abstract}
Resumo
Este trabalho teve como objetivo buscar evidências empíricas sobre o desempenho da análise gráfica considerando a lucratividade quando montadas em um sistema autônomo para o disparo de ordens de compra e venda de ações via homebroker na Bovespa, bem como apresentar uma metodologia modificada para o teste de Lorenzoni et al. (2007) para verificar a presença de conteúdo informacional na análise técnica. Este sistema autônomo consiste em uma rotina modificada do comportamento racional de compra constante em Arrow (1959), que visa ao lucro máximo e procura simular o comportamento humano como estratégia para o disparo de ordens. Foram testadas 352 combinações diferentes de possibilidades de ordens, quando acopladas ao comportamento racional para cada série testada. Os dados utilizados compreenderam as empresas constantes no índice FGV-100 (2009) e no Ibovespa (set./dez. de 2009) totalizando 129 companhias no período de 03/01/2000 até 30/09/2009. Os principais resultados mostram que bons desempenhos foram obtidos pelas médias móveis, seguidos das bandas de Bollinger e do histograma, que não se mostrou eficiente. As ordens foram contadas em quantidades. Foram encontrados comportamentos racionais de compra e venda com desempenho superior aos seus semelhantes, quando comparados em termos de lucratividade aos retornos obtidos pelo Ibovespa. A estatística de White (2000) e o teste modificado de Lorenzoni et al. (2007), proposto pelo autor, sugeriram a presença de conteúdo informacional para a maioria das séries testadas na amostra quando os gráficos indicaram a posição comprada, tanto para as séries originais quanto para os dados recriados a partir do modelo $\operatorname{AR}(1)-\mathrm{GARCH}(1,1)$.

Palavras-chave: Finanças. Análise gráfica. Comportamento racional. Lucros. Mercado de capitais.

\footnotetext{
1 Mestre em Administração - Finanças pela Universidade Federal do Rio Grande do Sul (UFRGS), doutorando em Administração - Finanças pela Universidade de Brasília (UnB). É Professor Assistente de Finanças da Universidade Federal de Uberlândia (UFU). Brasil. E-mail: jpietron@gmail.com

2 Mestre em Administração pela Universidade Federal do Rio de Janeiro (UFRJ), mestre (MSc) em Economia pela Universidade de Londres, Reino Unido, doutor (PhD) em Economia pela Universidade de Southampton, Reino Unido. É Professor Titular da Universidade de Brasília e Coordenador do Programa de Pós-Graduação em Administração e Vice-Diretor da Faculdade de Economia, Administração e Contabilidade. Brasil. E-mail: otavio@unb.br
} 


\begin{abstract}
The purpose of this paper aims to get empirical evidence about the performance of Technical analysis considering profitability when coupled on a stand-alone system to trigger orders of buy/selling shares via home broker at Bovespa, also present a modified methodology for the Lorenzoni et al. (2007) test to check the presence of informational content in technical analysis. This autonomous system consists of a modified routine of rational choice constant in Arrow (1959) which seeks maximum profit trying to simulate human behavior as a strategy for triggering orders. It was tested 352 different combinations of possible orders when attached to the rational behavior for each series tested. The data used were companies listed in FGV-100 index (theoretical portfolio of the year 2009) and Ibovespa (theoretical portfolio of Sept/Dez 2009) with a total of 129 companies from 3/01/2000 to 09/30/2009. The main results show that the best results were obtained by moving average followed by Bollinger bands and, finally the histogram, which has not proved by self efficient. Orders were counted in quantities. It was found rational choice behaviors of buy/sell with superior performance to its peers when compared in terms of profitability with the returns obtained by Ibovespa. The statistics of White (2000) and the modified test of Lorenzoni et al. (2007), proposed by the author, had suggested the presence of informational content for the majority of the series tested in the sample when the graphs indicated long position for both the original data and for the recreated data from the $A R(1)-G A R C H(1,1)$ model.
\end{abstract}

Keywords: Finance. Technical analysis. Rational choice. Profits. Capital markets.

\title{
1 INTRODUÇÃO
}

O debate sobre a busca da maximização da riqueza do investidor foi impulsionado no começo da década de 1950, com destaque para o trabalho de Markowitz (1952) na gestão de portfólios. Posteriormente, através do modelo CAPM, atribuído a Sharpe (1964), que veio a ser utilizado como modelo de previsão de retornos dos ativos. Esse modelo, aprimorado, por Ross (1976) gerou a teoria do preço arbitrado (APT). Um dos objetivos da construção destes modelos matemáticos foi o de sugerir valores teóricos das ações que refletiriam a realidade empresarial, calcados no desempenho dos preços históricos dos ativos.

Tradicionalmente, três técnicas são conhecidas e utilizadas para avaliar os ativos e fundamentar a emissão de ordens de compra e venda por parte dos investidores: a análise fundamentalista, análise gráfica e análise econométrica.

A análise gráfica, também conhecida como análise técnica, induz o investidor a escoIher seus ativos com base no formato dos gráficos gerados pelas cotações das ações. Algumas das técnicas utilizadas são os gráficos de médias móveis, o histograma de médias, as bandas de Bollinger, o índice de força relativa, o gráfico estocástico, os números de Fibonacci, o gráfico castiçal (candlestick), o suporte e a resistência etc.

Nesse sentido, a investigação de qual dos tipos gráficos adicionou mais valor em termos absolutos ao investidor motivou a proposta deste trabalho, em que os seguintes questionamentos foram feitos: para a obtenção de lucros nas ações negociadas na BOVESPA, qual técnica gráfica oferece os melhores retornos para o investidor que possui preferências racionais $(\succ)^{3}$ ? Quais comportamentos de compra obtiveram os melhores resultados?

Considerando os questionamentos levantados, o desenvolvimento deste trabalho justifica-se como ferramenta de auxílio aos investidores para a escolha do tipo de análise gráfica a ser utilizada como metodologia de canalização dos recursos a serem investidos no mercado acionário.

\footnotetext{
${ }^{3}$ A preferência racional será, de agora em diante, representado pelo símbolo $\succ$. Como exemplo, tem-se que $x \succ z$ deve ser interpretado como x é preferível em relação à z.
} 
Os objetivos principais deste estudo foram o de comparar e o de obter estatísticas a respeito da eficiência dos gráficos utilizados pelos investidores para sua utilização como fonte de informação nos investimentos em ações da BOVESPA, como fizeram Sullivan, Timmermann e White (1999). Também o de verificar a possibilidade de robotização das operações, sendo que as técnicas comparadas foram: os gráficos de médias móveis, as bandas de Bollinger e o histograma de médias.

Como objetivo secundário, este trabalho oferece uma visão empírica sobre o desempenho desses modelos, considerando uma rotina que representaria o comportamento de compra de um investidor com preferências racionais, como detalha Arrow (1959), com algumas modificações. A amostra utilizada compreendeu 129 empresas componentes dos índices FGV-100 e Ibovespa com memória de dados diários no período de 03/01/2000 até 30/09/2009.

Este trabalho está organizado da seguinte forma: no seção 2, é apresentada a fundamentação teórica que fornece um conjunto de estudos relacionados com o tema contemplado; na seção 3, encontra-se o método utilizado e as características relevantes adotadas para o comportamento de compra do investidor, bem como o detalhamento dos modelos, o processo de estimação e as estatísticas utilizadas para a validação dos dados; na seção 4, são apresentados os resultados obtidos com a pesquisa; na seção 5, é feito um resumo, em que são evidenciados os principais resultados e as conclusões deste trabalho.

\section{FUNDAMENTAÇÃO TEÓRICA}

A técnica da análise gráfica passou a ser amplamente explorada, principalmente após os trabalhos de Edwards e Magee (1966), Murphy (1986) e Treynor e Ferguson (1985). Apesar de a opinião científica ser fundamentada em razões sérias para sua não aceitabilidade, pois se baseiam em premissas fracas, como, por exemplo, a hipótese de que padrões passados selecionados irão se repetir no futuro (JEGADEESH, 2000), a análise gráfica pode ser tão importante quanto a análise fundamentalista ou econométrica para os investidores.

Um dos modelos mais conhecidos e disseminados, talvez por sua facilidade de aplicação, é o de médias móveis, que entrou para o meio acadêmico com um dos trabalhos pioneiros de Gartley (1935). Este tipo de técnica é baseado na elaboração de novas séries calculadas a partir dos dados originais, considerando médias de diferentes períodos passados para a montagem de estratégias de compra e venda.

Atualmente, a elaboração de novas séries para a execução dessa estratégia é facilitada pelo aparato computacional, que permite a geração de dados em períodos curtíssimos de tempo, como, por exemplo, o trabalho realizado por Sullivan, Timmermann e White (1999), que utilizou médias de 2, 3, 4,..., 300 dias. Estes modelos foram testados pela técnica de bootstrap, para que as melhores configurações fossem reutilizadas posteriormente para verificar a continuidade do desempenho e de seu poder preditivo.

Outros modelos gráficos utilizados são o cabeça-ombro, topos largos, topos triangulares e retangulares e topos duplos e fundos. Estes gráficos foram profundamente explorados por Lo, Mamaysky e Wang (2000), sendo estimados e suavizados com regressões estimadas com suavização Kernel (NADARAYA, 1964; WATSON,1964) para a formação dos modelos de previsão.

Os resultados mostraram que a grande maioria das técnicas empregadas podem ser utilizadas como fontes confiáveis de informações, principalmente para as ações da Nasdaq, embora os topos triangulares e retangulares não tenham oferecido resultados ótimos. Ao contrário desses resultados, Jegadeesh (2000) não encontrou nenhuma dessas regras gráficas como forma significante para obtenção de retornos diferentes de zero, com o uso da estatística -t. 
Sullivan, Timmermann e White (1999) também testaram algumas regras de filtragem, suporte e resistência, médias de volume balanceado, médias móveis e regras de alcance (Channel Breakouts). Um dos resultados encontrados mostrou que a análise de médias móveis com janelas curtas de tempo (2, 3, 4 e 5 dias) foram as que obtiveram os melhores indicadores de ajuste, de acordo com a obtenção de retornos médios. Porém não mantiveram seu desempenho nas séries projetadas.

Fock, Klein e Zwergel (2005) também não encontraram resultados significativos e diferentes do benchmark passeio aleatórios para gráficos do tipo castiçais na série intradiária (5 mim) do FDAX (DAX Futures German Stock Index) de 2002 a 2003.

\section{METODOLOGIA}

Para a realização deste trabalho, foram consideradas apenas as informações das cotações dos ativos como informações disponíveis no mercado, portanto, foram desenvolvidas considerando as hipóteses de eficiência fraca de mercado, conforme Fama (1970), já discutidas em Cowles (1933) e Samuelson (1965).

Sendo assim, a elaboração da pesquisa consistiu nas etapas discriminas a seguir:

1. Criação de rotinas por meio de linhas de comando nos softwares Excel e Matlab;

2. No Excel, foram criadas as rotinas do comportamento racional de compra modificado para todos os tipos gráficos e realizados os testes estatísticos de White (2000), Lorenzoni et al. (2007), qui-quadrado e as regras de filtragem, descritos, em detalhes, nas seções abaixo. Em Visual Basic (linguagem de programação do Excel), foi feita a integração entre Matlab e Excel para que os resultados das séries estimadas pudessem ser transferidos em conjunto para o Excel e para a adição do comportamento de compra sobre as séries criadas, com intuito de verificar a eficácia do comportamento dos dados na obtenção de resultados;

3. Com a integração pronta, todos os dados de output do Matlab foram importados para o Excel e a rotina do comportamento racional de compra foi acoplada nas séries geradas para os cálculos da obtenção do lucro máximo;

4. As séries foram comparadas de acordo com a obtenção de lucros e a aderência ao sistema de validação.

No total, 352 combinações de diferentes possibilidades de comportamento racional de compra/venda foram testadas, sendo 96 para os modelos de médias móveis, 140 para os modelos do histograma de média e 116 para as bandas de Bollinger para cada série de ativos da amostra.

Para todos os modelos, foi considerado o custo de corretagem em número de operações. Os modelos estimados foram acrescentados de mecanismos para iniciar o disparo de ordens. Estes mecanismos foram chamados de regras de filtragem e são detalhados a seguir. $\mathrm{O}$ software considera que o investidor inicial parte de um investimento fictício de $\$ 100,00$ e todo o lucro ou prejuízo é reinvestido, podendo ser comprado um lote fracionário. Compra e venda a descoberto não foram permitidos.

Para cada modelo gráfico escolhido, foi seguido um padrão de emissão de cada ordem, conforme as exigências de cada modalidade, sendo vários padrões criados aleatoriamente na tentativa de encontrar algum padrão significativo. Considerando o caso do gráfico de médias móveis, por exemplo, foi executada a compra quando a linha de tendência para os dados de média móvel pequena cruzaram a linha de média longa para baixo. Estes padrões são detalhados no item 3.1. Esta rotina manteve a posição comprada até que um sinal de venda fosse disparado. 


\subsection{Os modelos gráficos}

Modelos gráficos simples como o de médias móveis foram testados por autores renomados, como Brock, Lakonishok e LeBaron (1992), Sullivan, Timmermann e White (1999), Wang e Chan (2007), Batchelor e Kwan (2007) etc.

Neste trabalho, para o gráfico de médias móveis, foi utilizada uma metodologia modificada de Brock, Lakonishok e LeBaron (1992), que utiliza dois sinais de compra $\left(P_{t}>M A_{T, L} \quad\right.$ e, $\mathrm{P}_{t-1}<M A_{t-1, L} \quad$ com, $\quad \mathrm{MA}_{t, L}=\frac{1}{L} \sum_{j=0}^{L-1} P_{T-j}$ resumidamente, onde P é o preço da ação, e MA a média móvel), com a utilização de 2 ou 3 médias móveis simultâneas, conforme a figura 1. Esta configuração, com três médias, foi composta para alguns dos modelos de comportamento testados. Essas médias se enquadraram em uma das seguintes categorias: média curta, intermediária ou longa, por simplificação 7, 21 e 32 dias. As subdivisões para médias curtas, intermediárias e longas foram definidas no comportamento racional de compra/venda. Dessa forma, uma média móvel de 16 dias anteriores pode ser interpretada como média curta, intermediária ou longa, dependendo do modelo proposto.

O cálculo das médias móveis foi feito seguindo

a equação $E\left(P_{n}\right)=\frac{P_{n-i}+P_{n-i-2}+\ldots P_{1}}{n}=\frac{\sum_{i=1}^{n} P_{t-i}}{n}$

com médias variando entre 2 e 32 dias.

A estratégia de compra e venda foi efetuada respeitando-se as seguintes condições: sinais de compra quando a média intermediária $\left(E\left(P_{I}\right)\right)$ cruza a média curta $\left(E\left(P_{c}\right)\right)$ para baixo e quando a média longa ( $E\left(P_{L}\right)$ ) cruza a média intermediária para baixo. $f$ são as regras de filtragem apresentadas no item 3.2. Resumo:

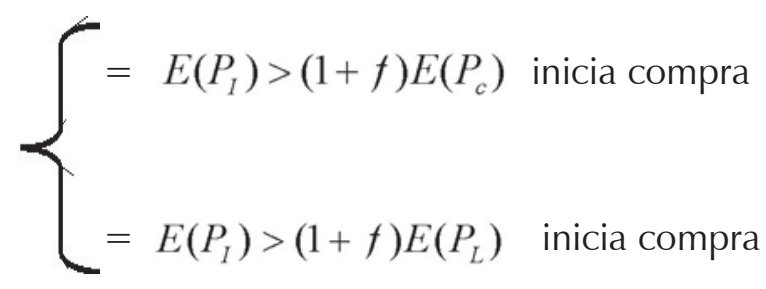

Sinais de venda: quando a média intermediária cruza a média curta para cima e quando a média longa cruza a média intermediária para cima.

$$
\left\{\begin{array}{l}
=E\left(P_{c}\right)>(1+f) E\left(P_{I}\right) \text { inicia venda } \\
=E\left(P_{L}\right)>(1+f) E\left(P_{I}\right) \text { inicia venda }
\end{array}\right.
$$


Nestes termos, as seguintes ordens de compra/venda foram adotadas e consideradas como comportamento racional modificado de compra em conjunto com as regras de filtragem:

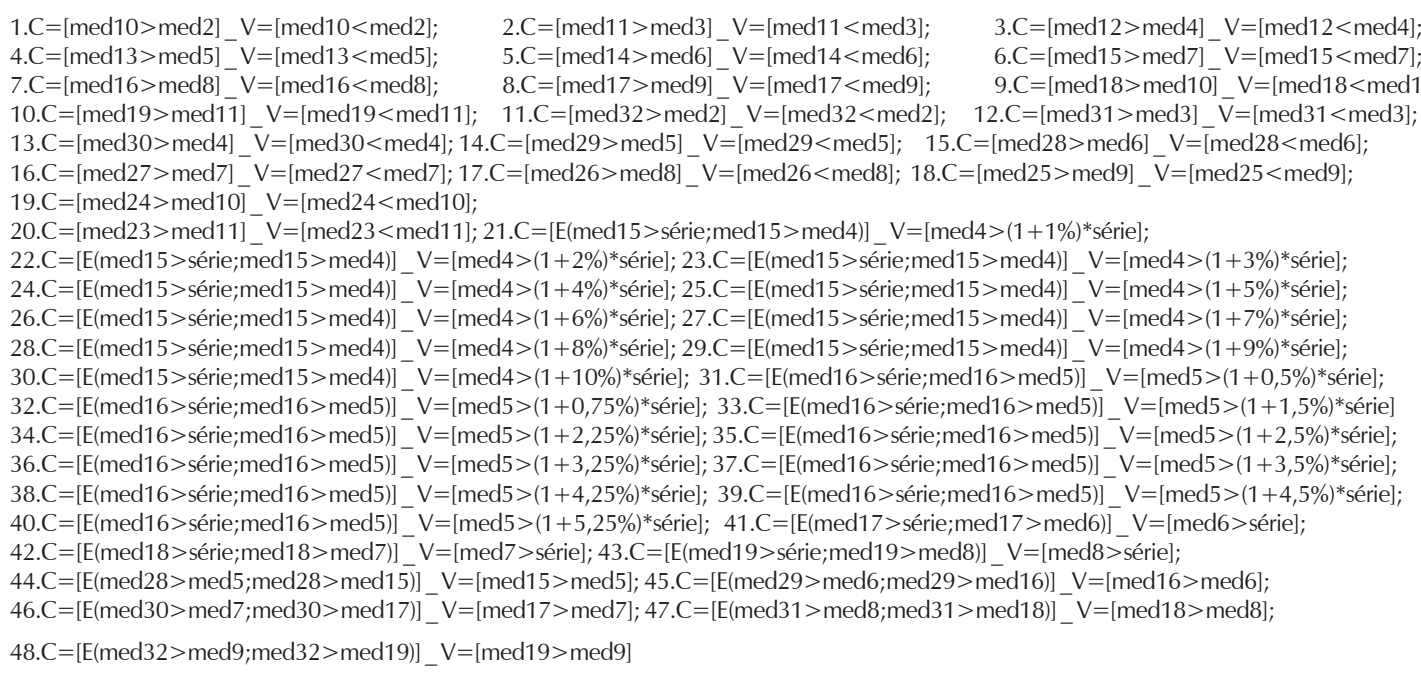

A modificação ocorrida é obtida na medida em que trata a maximização da utilidade representada apenas pela maximização da riqueza e não da utilidade do investimento em si. Nos comportamentos, $\mathrm{C}$ representa a ordem de compra, $\mathrm{V}$ a ordem de venda, $\operatorname{med}_{i}$, $\operatorname{com} i=$ $[1,2, \ldots 32]$, representa a média móvel de $i$ dias da série original, série é a série de dados original e $E$ é um operador lógico que indica que as $n$ (número de condições) condições devem ser satisfeitas para que a ordem seja disparada. As combinações somaram 48 tipos de diferentes ordens de compra e 48 de venda, totalizando 96 combinações para o disparo das ordens.

Como exemplo, o comportamento $(1 . C=[\operatorname{med} 10>\operatorname{med} 2]-V=[\operatorname{med} 10<\operatorname{med} 2])$ é traduzido da seguinte forma para a linguagem de operações: o software irá comprar ações da empresa quando a série criada a partir da média dos últimos 10 dias for maior do que a série criada a partir da média dos 2 últimos dias de pregão. A venda foi realizada quando a situação oposta aconteceu.

Vale lembrar que, para todas as estratégias, o comportamento racional de compra e venda assumiu uma estratégia do tipo buy and hold até que a figuração do comportamento se descaracterizou. Neste momento, o software disparou a ordem de venda.

Outra técnica utilizada se refere ao histograma de médias. Os cálculos das médias foram efetuados com a especificação:

$E\left(\operatorname{Re} t_{n}\right)=\frac{\operatorname{Re} t_{n-i}+\operatorname{Re} t_{n-i-2}+\ldots . \operatorname{Re} t_{1}}{n}=\frac{\sum_{i=1}^{n} \operatorname{Re} t_{t-i}}{n}$, agrupados com janela móvel variando de $\operatorname{dias}\left(E\left(\operatorname{Re} t_{n}\right)\right)$.

A estratégia de compra e venda, para o histograma de médias, foi efetuada respeitandose as seguintes condições: sinais de compra, quando a média intermediária $\left(E\left(\operatorname{Re} t_{n}\right)\right)$ estiver 
abaixo da E(retsérie) , que é a média dos retornos para todo o período estudado. Resumo:

$$
\left\{=E\left(\operatorname{Re} t_{n}\right)<(1+f) E(\text { retsérie })\right. \text { inicia compra }
$$

Sinais de venda: quando a média intermediária $\left(E\left(P_{n}\right)\right)$ estiver acima de zero.

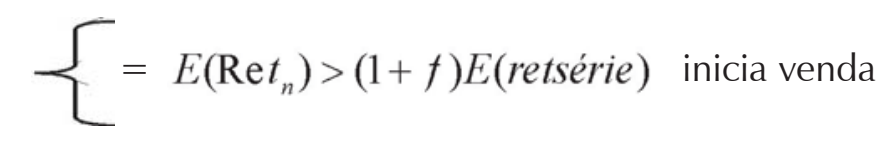

Para este modelo, as seguintes ordens de compra/venda foram adotadas:

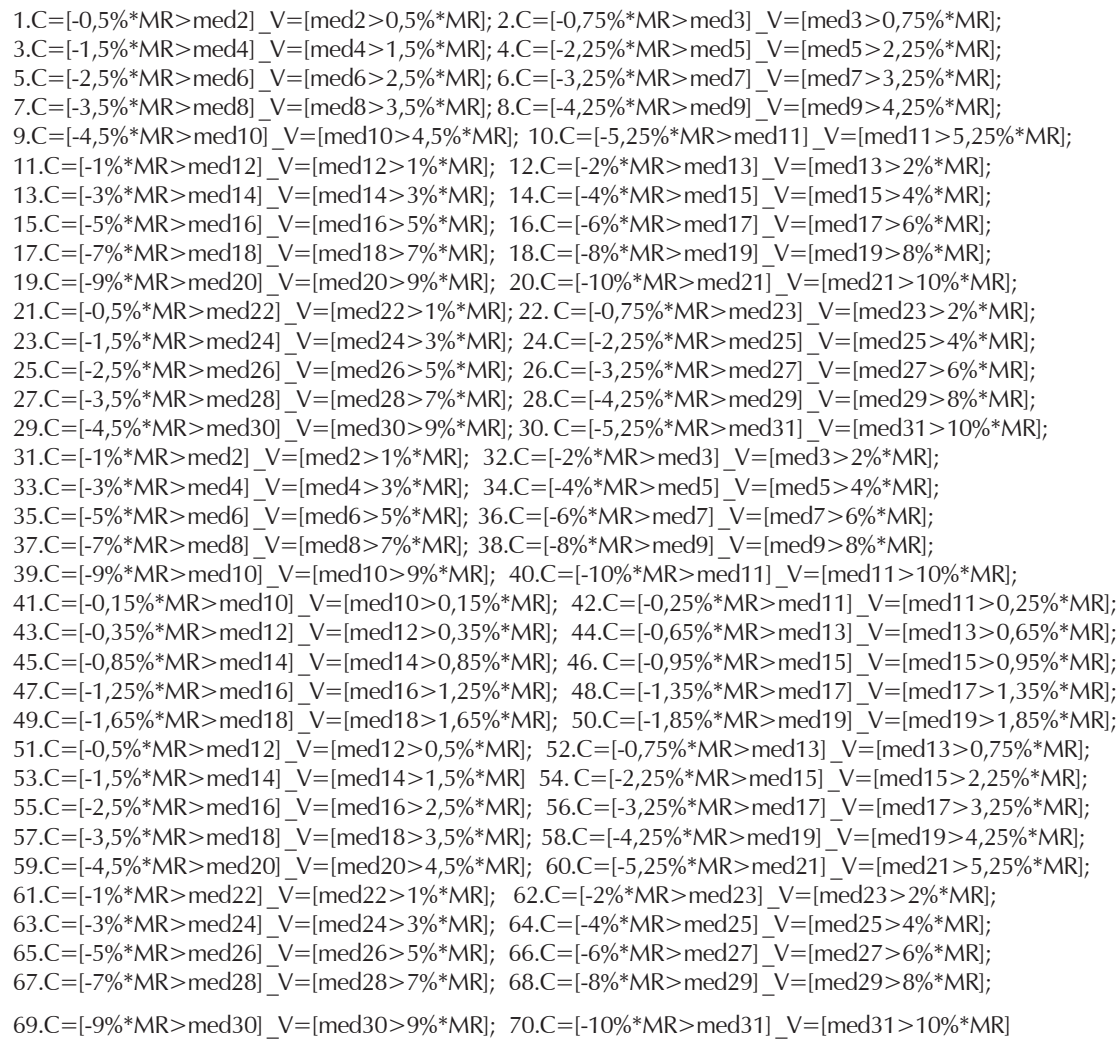

Sendo: $C=$ compra, $V=$ venda, $M R=$ média dos retornos da série original, med $_{i}$, com $i=[2,3, \ldots 32]$, representa a média móvel de $i$ dias da série de retornos da série original. As combinações somaram 70 tipos de diferentes ordens de compra e 70 de venda, totalizando 140 combinações para o disparo das ordens.

A última estratégia gráfica testada foram as bandas de Bollinger, sendo que o cálculo das bandas foi feito a partir das fórmulas abaixo: 


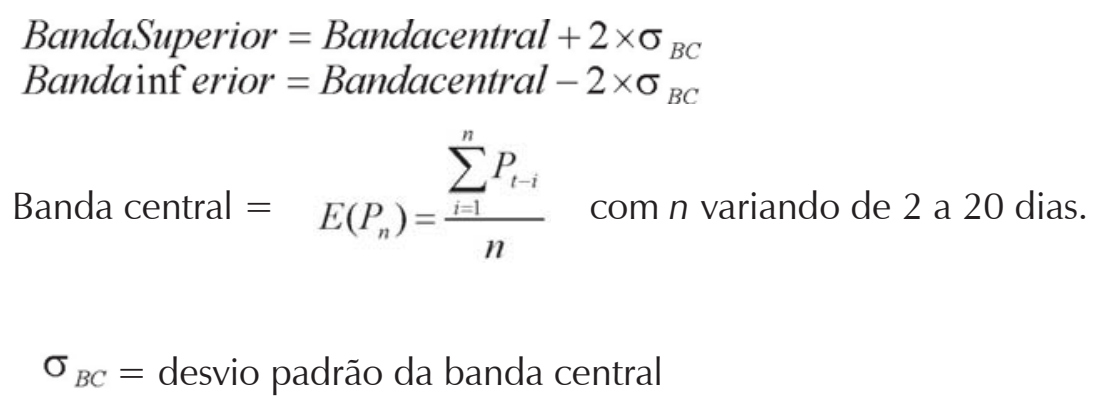

A estratégia de compra e venda, nesse caso, foi efetuada respeitando-se as seguintes condições: sinais de compra, quando a cotação real da ação cruza a banda central para baixo e quando a cotação se aproxima da banda inferior, respeitando os filtros de compra.

$$
\begin{aligned}
& \left\{P_{i}>(1+f) E\left(P_{B C}\right)=\right.\text { inicia compra } \\
& P_{B C}=\text { Preço da banda central }
\end{aligned}
$$

Sinais de venda: quando a cotação cruza a banda central para cima e quando a cotação se aproxima da banda superior, respeitando os filtros de venda.

$$
P_{i}>(1+f) E\left(P_{B C}\right)=\text { inicia venda }
$$

Para as bandas de Bollinger, as seguintes ordens foram disparadas:

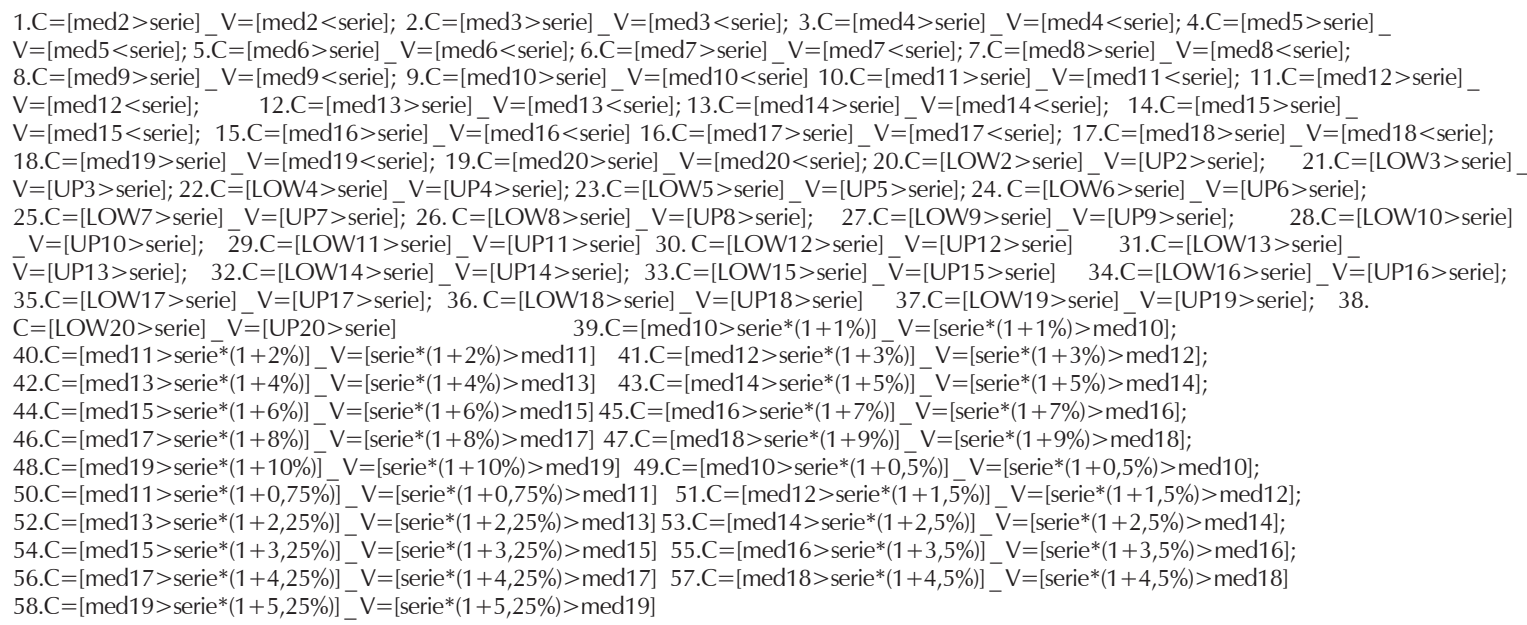

Sendo: $C=$ compra, $V=$ venda, $M R=$ média dos retornos da série original, med $_{i}$, com $i=[1,2, \ldots 20]$, representa a média móvel de $i$ dias da série de retornos da série original, série é a série de dados original, $\mathrm{LOW}_{\mathrm{s}}$ é a banda inferior, $\operatorname{com} s=[1,2, \ldots, 20]$, UP $\mathrm{r}$ é a banda superior, com $r=[1,2, \ldots, 20]$. As combinações somaram 58 tipos de diferentes ordens de compra e 58 de venda, totalizando 116 combinações para o disparo das ordens. 


\subsection{As regras de filtragem}

As regras de filtragem (f) foram utilizadas para garantir que as ordens de compra e venda fossem efetivamente realizadas durante várias alternativas. Assim, se considerarmos que $X$ representa o preço de um ativo, então, para que a posição de compra seja iniciada, deverá ocorrer uma variação $C$ positiva ou negativa $(X \times C)$ na cotação do ativo.

Estratégia igual será utilizada para se iniciar a liquidação das posições compradas. Logo, quando $X$ sofrer alguma variação negativa ou positiva $V(X \times V)$, será iniciada a venda das posições que estão encarteiradas. Assim,

$C=$ mudança no preço requerida para que se inicie a compra dos ativos;

$V=$ mudança no preço requerida para que se inicie a liquidação dos ativos em carteira;

$f=$ regra de filtragem para compra e venda para médias móveis $[0,005 ; 0,0075 ; 0,015 ;$

0,0225;0,025;0,0325;0,035;0,0425;0,045;0,0525;0,01;0,02;0,03;0,04;0,05;0,06;0,07;0,08;0,09;0,1].

Para o histograma de médias, as regras de filtragem foram $[0,005 ; 0,0075 ; 0,015 ; 0,0225 ; 0,025 ; 0,0325 ; 0,035 ; 0,0425 ; 0,0045 ; 0,0525 ; 0,0015 ; 0,0025 ; 0,0035 ; 0,0065$; 0,$0085 ; 0,0095 ; 0,0125 ; 0,0135 ; 0,0165 ; 0,0185 ;-0,005 ;-0,0075 ;-0,0015 ;-0,0225 ;-0,025 \quad ;-0,0325 ;-$ 0,$035 ;-0,0425 ;-0,0045 ;-0,0525 ; 0,01 ; 0,02 ; 0,03 ; 0,04 ; 0,05 ; 0,06 ; 0,07 ; 0,08 ; 0,09 ; 0,1 ;-0,0015 ;-0,0025 ;-$ 0,$0035 ;-0,0065 ;-0,0085 ;-0,0095 ;-0,0125 ;-0,0135 ;-0,0165 ;-0,0185 ;-0,01 ;-0,02 ;-0,03 ;-0,04 ;-0,05 ;-0,06 ;-$ $0,07 ;-0,08 ;-0,09 ;-0,1]$, sendo multiplicados pela média dos retornos das séries.

\subsection{Validação da análise gráfica}

A validação das estratégias gráficas foi feita com a utilização da metodologia proposta por Lorenzoni et al. (2007), com a introdução de limitações ao processo de reprodução das séries estimadas pelo modelo $\mathrm{AR}(1)-\mathrm{GARCH}(1,1)$ e posterior comparação através do teste quiquadrado. 
O modelo de Lorenzoni et al. (2007) pode ser descrito pela figura 1.
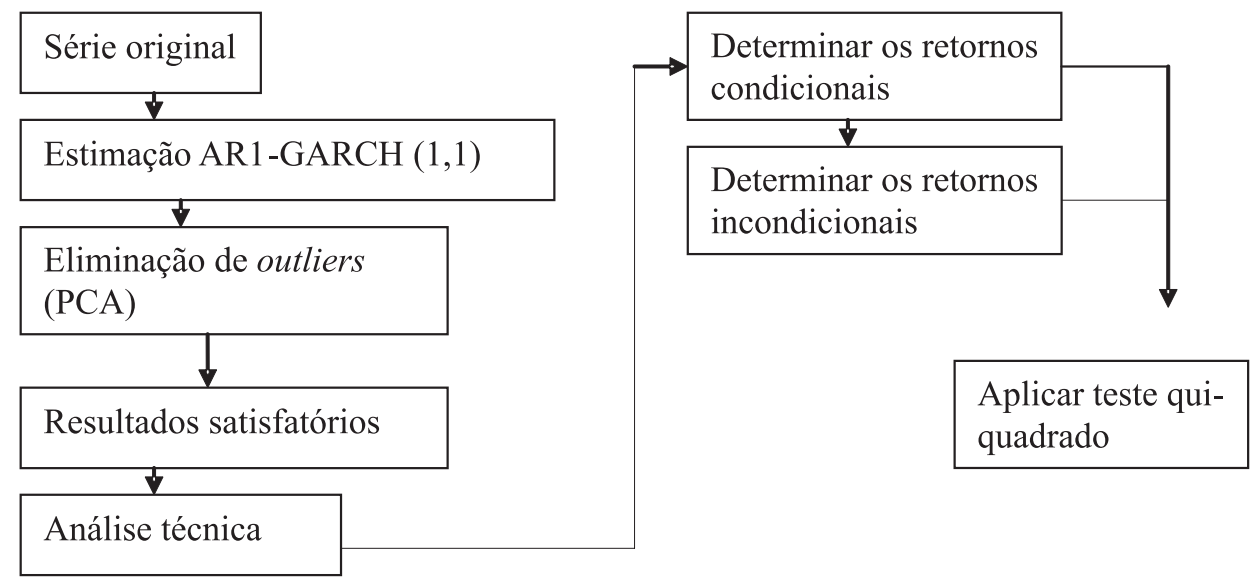

Figura 1 - Modelo de Lorenzoni et al. (2007)

Esse modelo propõe a criação de uma série nova, a partir da série original, com o modelo AR(1)-GARCH(1,1) e a separação dos outliers com uma análise PCA (principal components analysis) para que a série criada seja a mais próxima possível da original. Logo, o teste procura evidenciar se há a presença de heterogeneidade, com uma comparação dos retornos condicionais (dias de negociação onde foram evidenciados padrões de comportamento procurados) aos retornos incondicionais (retornos de todas as séries ou retornos do lbovespa). Caso o teste qui-quadrado seja significativo, então os padrões comparados apresentam conteúdo informacional.

As limitações propostas neste trabalho são referentes aos limites altos e baixos (upper and lower bounds), com a utilização do algoritmo oferecido no software Matlab ${ }^{\circledR}$. A introdução dessa nova proposta eliminaria a necessidade de realização da análise PCA sugerida pelos autores para a separação dos outliers, oferecendo a criação de séries semelhantes às originais (médiavariância), com a possibilidade de automatização dos cálculos. Graficamente a nova proposta seria a apresentada na figura 2 .
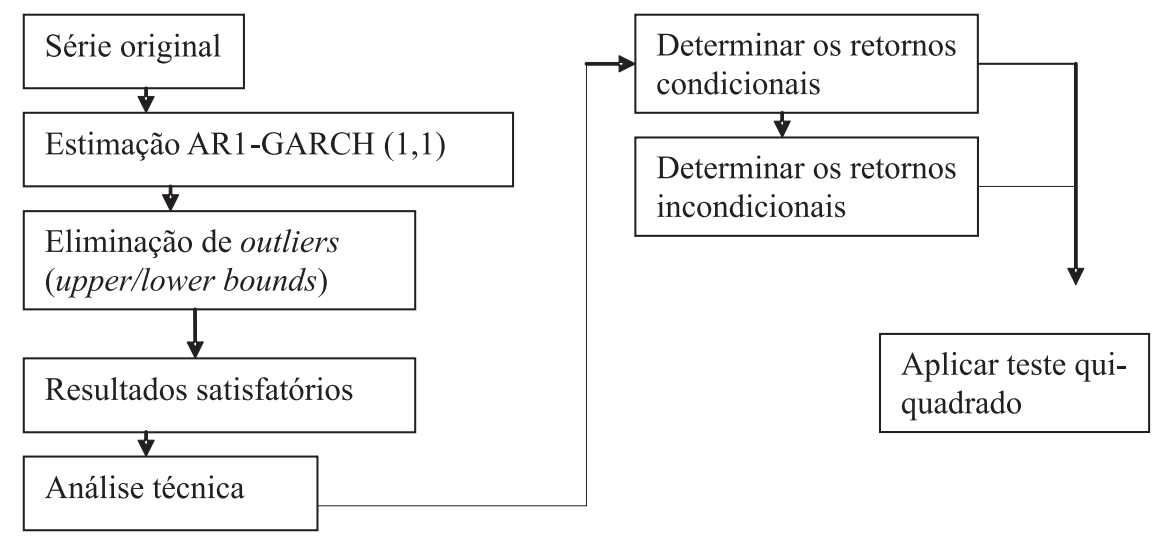

Figura 2 - Modelo proposto pelo autor 
A limitação imposta se refere aos parâmetros estimados com

$\alpha_{i}>0, i=1,2, \ldots, q ; \beta_{\mathrm{j}}>0, j=1,2, \ldots, p$ e $\alpha_{i}+\beta_{j}<1$ e o termo constante, $\alpha_{0}>0$ sendo, tipicamente $\alpha_{0}=2 e^{-7}$ com limitação de 3 desvios padrões da própria série préanalisada. Nesse caso, a nova série criada não conterá valores estimados pelo modelo $A R(1)$ GARCH $(1,1)$ que contenham inovações superiores a 3 desvios em relação à média da série original.

Após essa avaliação, foi aplicado o teste de realidade proposto por White (2000), e os resultados foram comparados.

Para a análise do histograma de médias e de bandas de Bollinger, utilizamos a comparação da estratégia com um benchmarck de mercado (Ibovespa), como sugerido por White (2000). Assim, para verificar se as estratégias testadas obtiveram retornos anormais positivos ou negativos, o teste de White foi aplicado com $H_{0}$ considerando a não presença de retornos anormais, como fizeram Bajgrowicz e Scaillet (2008) e Saffi (2003). Logo, as hipóteses foram configuradas da seguinte forma: $H_{0 k}=0$ e $H_{1 k}: i a_{k}>0$ ou $a_{k}<0$.

O BRC (Bootstrap Reality Check) proposto por White (2000) procura solucionar os problemas provocados pela escolha ao acaso dos modelos testados (data snooping), com a

$$
\mathrm{H}_{0 \mathrm{k}}=\max _{\mathrm{k}=1, \ldots, \mathrm{N}} \alpha \mathrm{K} \leq 0 \quad \text { para as análises testadas. }
$$

A especificação do teste considera que se $n$ for o tamanho da série utilizada a ser testada, produz-se um vetor $E\left(f^{*}\right)$ de tamanho $/ \times 1$, onde $f^{*}=f\left(Z, B^{*}\right)$ é um vetor $/ \times 1 \mathrm{com}$ elementos $f_{K}^{*}=f_{K}\left(Z, B^{*}\right)$ onde $Z$ e $B^{*} \equiv p \lim B$, e $Z$ consiste em vetores de variáveis dependentes de $Y$ regredido contra $X$. A base do teste se dá no vetor $I \times 1$ obtida por

$$
\bar{f} \equiv n^{-1} \sum_{t=1}^{n} \hat{f} \quad \text { e } \quad \hat{f} \equiv f\left(Z_{t}, \hat{B}_{t}{ }^{*}\right) \quad \text {, sob a hipótese nula de que } H_{0}: E\left(f^{*}\right) \leq 0
$$

Logo, para testar a hipótese de que uma estratégia é superior ao benchmark utiliza-se,

$$
\hat{f}_{t+1}=\ln \left[1+y_{t+1} S_{1}\left(X_{1, t+1}, B_{1}^{*}\right)\right]-\ln \left[1+y_{t+1} S_{0}\left(X_{0, t+1}, B_{0}{ }^{*}\right)\right] \quad \text { onde } y_{t+1} \quad \text { são os retornos }
$$

diários, e $\mathrm{S}_{0}$ e $\mathrm{S}_{1}$ são funções que convertem os indicadores de análise gráfica ( $X_{0, t+1}$ e $X_{1, t+1}$ ) e os parâmetros ( $B_{0} *$ e $B_{1} *$ em posições de compra e venda. As funções puderam assumir três valores: 1(vendido), 0 (não comprar e não vender) e 1 (comprado). Nesse sentido, $\left(B_{0} *\right.$ e $B_{1} *$ ) são estabelecidos a priori e não precisam ser estimados. Assim, a hipótese que testa o modelo com melhor performance sobre o benchmark de mercado é dada por. 


$$
\mathrm{H}_{0 \mathrm{k}}=\max _{\mathrm{k}=1, \ldots, \mathrm{N}} \mathrm{E}\left(\mathrm{f}_{\mathrm{k}}^{*}\right) \leq 0
$$

\subsection{Os modelos gráficos simulados}

A partir da criação das séries propostas por Lorenzoni et al. (2007) com a reprodução das séries estimadas pelo modelo $\operatorname{AR}(1)-\mathrm{GARCH}(1,1)$ criando-se séries simuladas, todos os modelos gráficos (médias móveis, bandas de Bollinger e histograma de médias) foram rodados em sua integralidade para as séries simuladas; o mesmo procedimento para o comportamento racional de compra e venda foi utilizado.

Nesse sentido, para a análise gráfica, dois conjuntos de dados foram testados: os dados originais e as séries simuladas pelo modelo $\operatorname{AR}(1)-\mathrm{GARCH}(1,1)$, com as limitações impostas pelo autor deste trabalho. Posteriormente, os resultados foram comparados quanto aos lucros.

\subsection{Amostra}

A amostra foi composta pelas séries de preços e índices financeiros das empresas componentes do índice Ibovespa, carteira teórica referente aos meses de set./dez. de 2009, e do índice FGV-100, carteira teórica referente ao ano de 2009 (05/10/2009), perfazendo um total de 129 empresas. Na tabela 1, são discriminadas as empresas componentes da amostra.

\begin{tabular}{|l|l|l|l|l|l|l|l|l|}
\hline \multicolumn{7}{|c|}{ Código } \\
\hline AVIL3 & AGRO3 & CYRE3 & FJTA4 & LIGT3 & ODPV3 & SDIA4 & TCSL4 & WEGE3 \\
\hline GETI4 & BRKM5 & DASA3 & FFTL4 & RENT3 & OHLB3 & SMTO3 & TOTS3 & \\
\hline ALLL11 & BRFS3 & DROG3 & GFSA3 & LOGN3 & PCAR5 & SATI3 & TBLE3 & \\
\hline ALPA4 & BISA3 & DURA4 & GGBR4 & LAME4 & PDGR3 & CSNA3 & TRPL4 & \\
\hline AMBV4 & CCRO3 & ELET3 & GOAU4 & LREN3 & PRGA4 & SLCE3 & TRIS3 & \\
\hline ABNB3 & CLSC6 & ELET6 & GOLL4 & MDIA3 & PRGA3 & CRUZ3 & UGPA4 & \\
\hline AMIL3 & CMIG4 & ELPL6 & CGRA3 & MAGS5+MAGG3 & PETR3 & SUZB5 & UNIP6 & \\
\hline ARCZ6 & CESP6 & EMBR3 & GRND3 & POMO4 & PETR4 & TAMM4 & UOLL4 & \\
\hline BTOW3 & COCE5 & ENBR3 & GUAR3 & MRFG3 & PLAS3 & TGMA3 & USIM5 & \\
\hline BVMF3 & CGAS5 & ENGI4 & GVTT3 & MMXM3 & POSI3 & TNLP3 & USIM3 & \\
\hline BBRK3 & CNFB4 & EQTL11+EQTL3 & IGTA3 & MPXE3 & PRVI3 & TNLP4 & VCPA4 & \\
\hline BBDC4 & CPLE6 & ESTC11+ESTC3 & ROMI3 & MRVE3 & RAPT4 & TMAR5 & VALE5 & \\
\hline BRAP4 & CSAN3 & ETER3 & ITSA4 & MULT3 & RDCD3 & TLPP4 & VALE3 & \\
\hline BBAS3 & CPFE3 & EZTC3 & JBSS3 & NATU3 & RDNI3 & TEMP3 & VIVO4 & \\
\hline BRTP4 & CSNA3 & FHER3 & JHSF3 & NETC4 & RSID3 & TEND3 & VCPA4+VCPA3 & \\
\hline BRTO4 & CCPR3 & FESA4 & KLBN4 & BNCA3 & SBSP3 & TCSL3 & VCPA4+FIBR3 & \\
\hline
\end{tabular}

Tabela 1 - Empresas componentes da amostra

Fonte: Bovespa. Disponível em: < http://www.bovespa.com.br/Principal.asp>. e FGV < http://www2.fgv.br/ dgd/asp/index.asp >. Acesso em: 05 out. 2009. 
Os cálculos foram realizados em séries diárias. A base de dados utilizada compreendeu o período de 03/01/00 até 30/09/09, em um total de 2338 dias ou, aproximadamente, 40 trimestres.

\section{Análise dos resultados}

Nesta seção, serão apresentados os principais resultados encontrados pelos softwares na obtenção de lucros pelas estratégias de compra e venda adotados como comportamento racional do investidor.

As tabelas com os resultados completos são apresentadas em Pietro Neto (2011) para todas as empresas, logo, os resultados apresentados neste trabalho são resumos dos principais encontrados em extensas tabelas.

Para a análise de lucros, deve-se ter em mente um investimento aleatório feito no índice Ibovespa. Para isso, foram calculados os rendimentos de $\$ 100$ aplicados para todo o período e de forma anual, conforme a tabela 2.

Para o cálculo dos rendimentos, foram utilizadas duas metodologias: a primeira, através do cálculo do lucro por log retornos, em que o investimento final é obtido através da equação $=$ inv.inicial $*\left(1+\mathrm{LN}\left(\frac{\mathrm{ibov}_{\mathrm{df}}}{\mathrm{ibov}_{\mathrm{di}}}\right)\right)$ onde $d f$ e di são os pontos do lbovespa na data final e na data inicial; na segunda, o investimento final para o juro bancário ou juros compostos foi obtido com a seguinte expressão $=$ inv.inicial $* \exp \left(\mathrm{LN}\left(\frac{\mathrm{ibov}_{\mathrm{df}}}{\mathrm{ibov}_{\mathrm{di}}}\right)\right)$.

\begin{tabular}{|r|r|r|r|r|}
\hline \multirow{2}{*}{ Data inicial } & Data final & $\begin{array}{l}\text { Investimento } \\
\text { inicial R\$ }\end{array}$ & $\begin{array}{l}\text { Investimento final } \\
\text { R\$ (log retorno) }\end{array}$ & $\begin{array}{l}\text { Investimento final R\$ (juro } \\
\text { bancário) }\end{array}$ \\
\hline $\mathbf{0 3 / 0 1 / 0 0}$ & $\mathbf{3 0 / 0 9 / 0 9}$ & $\mathbf{1 0 0}$ & $\mathbf{1 6 5 , 4 8 1 8 1 7 4}$ & $\mathbf{1 9 2 , 4 7 9 2 5 0 9}$ \\
\hline $03 / 01 / 00$ & $02 / 01 / 01$ & 100 & 84,80293551 & 85,90134968 \\
\hline $02 / 01 / 01$ & $02 / 01 / 02$ & 100 & 81,0796777 & 82,76182985 \\
\hline $02 / 01 / 02$ & $02 / 01 / 03$ & 100 & 69,78011098 & 73,91910324 \\
\hline $02 / 01 / 03$ & $02 / 01 / 04$ & 100 & 157,5865853 & 177,8669927 \\
\hline $02 / 01 / 04$ & $03 / 01 / 05$ & 100 & 108,4536488 & 108,821255 \\
\hline $03 / 01 / 05$ & $02 / 01 / 06$ & 100 & 117,8173819 & 119,5033022 \\
\hline $02 / 01 / 06$ & $02 / 01 / 07$ & 100 & 127,9660108 & 132,2680167 \\
\hline $02 / 01 / 07$ & $02 / 01 / 08$ & 100 & 129,3921091 & 134,1678029 \\
\hline $02 / 01 / 08$ & $30 / 09 / 09$ & 100 & 88,60335742 & 89,22879133 \\
\hline
\end{tabular}

Tabela 2 - Rendimentos Ibovespa 
Observa-se, para fins de comparação, que o rendimento médio de um investimento feito no Ibovespa no período de dez anos foi de $65 \%$, aproximadamente, como pode ser visto na primeira linha da tabela acima.

Na tabela 3, são apresentados os lucros máximos obtidos pelos gráficos. A coluna MIN apresenta os lucros mínimos para o ativo em \$; a coluna MAX apresenta o lucro máximo obtido em \$; № ORDENS é a quantidade de ordens de compra e venda disparadas; MOD é o comportamento racional de compra e venda que gerou os resultados; Gráfico se refere a médias móveis; graficosim, a médias móveis simuladas; bolling e bollingsim se referem asa bandas de Bollinger e Bollinger simuladas; histmed e histmedsim a histogramas de médias originais e simulados.

$\mathrm{Na}$ análise de lucros, os dados que chamaram a atenção foram os lucros obtidos com a utilização das estratégias de compra e venda que estiveram muito acima do benchmark utilizado.

Os números impressionam pela magnitude dos lucros obtidos: o ativo MAGS5/3 obteve um aumento próximo a $6200 \%$ sobre o investimento inicial. Quando considerado o período de análise, coluna MAX, nota-se que um investimento aleatório no Ibovespa produziu aproximadamente $65 \%$. O modelo de comportamento racional que obteve este lucro foi o 30. C $=[$ LOW12>serie $]$ _ V = [UP12>serie $]$.

Certamente, este resultado está muito acima dos outros obtidos, o que poderia sugerir um comportamento outlier, no entanto, os resultados que o seguem estão muito acima dos rendimentos oferecidos pelo Ibovespa.

Outro dado que chama atenção na tabela 3 é a quantidade de vezes em que as séries recriadas a partir das séries originais atingem os lucros máximos em relação às séries originais. Esta tabela sugere que, aproximadamente, $70 \%$ das séries recriadas atingem melhores desempenhos do que as séries originais. Vale lembrar que apenas o sinal de compra/venda das séries simuladas foi utilizado, sendo que a compra/venda foram efetuadas no mesmo dia e a preços da série original. Nesse sentido, a utilização de processos estocásticos deve ser mais bem estudada para os próximos trabalhos, e a utilização do comportamento racional deve acompanhar esta melhoria.

Em 40 ativos, aproximadamente, com lucro máximo, houve uma predominância de modelos de médias móveis, nas séries originais e simuladas, e de bandas de Bollinger, nas séries simuladas. A presença de grandes lucros para gráficos de histogramas de médias, com as séries originais ou simuladas, não foi evidenciada. A tabela 3 apresenta os 20 melhores resultados. 


\begin{tabular}{clcccccc}
\hline & & MIN & № ORDENS & MOD & MAX & № ORDENS & MOD \\
\hline MAGS5+MAGG3 & Bollingsim & 3903,05 & 28 & 18 & $\mathbf{6 2 1 1 , 4 9 1}$ & 102 & $\mathbf{3 0}$ \\
ROMI3 & Gráfico & 78,51885 & 96 & 12 & $\mathbf{4 3 0 6 , 3 7 6}$ & 60 & $\mathbf{4 7}$ \\
LREN3 & Gráfico & 178,0812 & 48 & 11 & $\mathbf{4 1 8 0 , 8 8 3}$ & 52 & $\mathbf{4}$ \\
DROG3 & Gráfico & 37,76132 & 40 & 20 & $\mathbf{3 8 5 8 , 5 4 9}$ & 22 & $\mathbf{2}$ \\
RAPT4 & Gráfico & 48,04566 & 82 & 17 & $\mathbf{3 1 4 6 , 3 9 4}$ & 52 & $\mathbf{2}$ \\
CSNA3 & Gráfico & 96,05446 & 160 & 7 & $\mathbf{2 6 4 7 , 4 8 1}$ & 62 & $\mathbf{9}$ \\
RAPT4 & Graficosim & 92,27194 & 8 & 42 & $\mathbf{2 5 9 1 , 4 4 2}$ & 82 & $\mathbf{2 7}$ \\
CSNA3 & Gráfico & 96,59475 & 128 & 11 & $\mathbf{2 5 1 6 , 1 5 2}$ & 66 & $\mathbf{4 4}$ \\
AVIL3 & Graficosim & 49,53331 & 34 & 40 & $\mathbf{2 5 0 5 , 3 7 4}$ & 74 & $\mathbf{3 4}$ \\
TRPL4 & Bollingsim & 1248,347 & 34 & 2 & $\mathbf{2 4 1 3 , 5 2 2}$ & 148 & $\mathbf{3 2}$ \\
TRPL4 & Gráfico & 100 & 2 & 28 & $\mathbf{2 3 9 1 , 4 9 8}$ & 62 & $\mathbf{4}$ \\
TRPL4 & Gráfico & 100 & 2 & 28 & $\mathbf{2 3 3 3 , 8 6 5}$ & 64 & $\mathbf{1}$ \\
DROG3 & Graficosim & 84,72962 & 92 & 42 & $\mathbf{2 3 3 0 , 0 4 7}$ & 54 & $\mathbf{4 7}$ \\
AVIL3 & Gráfico & 120,737 & 82 & 46 & $\mathbf{2 2 7 3 , 8 3 8}$ & 78 & $\mathbf{4 7}$ \\
GOAU4 & Gráfico & 100 & 2 & 28 & $\mathbf{2 2 4 4 , 1 3 5}$ & 66 & $\mathbf{4 7}$ \\
FESA4 & Gráfico & 32,95367 & 88 & 13 & $\mathbf{2 0 9 4 , 4 3}$ & 62 & $\mathbf{4}$ \\
WEGE3 & Gráfico & 100 & 2 & 30 & $\mathbf{2 0 9 4 , 3 1}$ & 42 & $\mathbf{4 4}$ \\
MAGS5+MAGG3 & Gráfico & 39,81982 & 96 & 12 & $\mathbf{1 9 8 5 , 3 9}$ & 24 & $\mathbf{3}$ \\
RSID3 & Graficosim & 21,9569 & 202 & 3 & $\mathbf{1 9 5 9}$ & 62 & $\mathbf{3 0}$ \\
CGRA3 & Bollingsim & 677,8424 & 18 & 38 & $\mathbf{1 9 4 4 , 1 7 1}$ & 32 & $\mathbf{1 9}$ \\
\hline
\end{tabular}

Tabela 3 - Lucro máximo - gráficos

Quanto ao número de ordens disparadas, a tabela acima mostra que, de forma aproximada, em média 64 ordens de compra/venda foram feitas no horizonte de 10 anos, o que gera, aproximadamente, 7 ordens anuais.

Com relação aos modelos de comportamento racional de compra, o destaque cabe aos modelos 2, 30, 47 e 44, sendo os mais promissores entre as estratégias testadas. Estes modelos aparecem, com frequência, nas séries de maiores lucros, tanto para as séries originais quanto para as simuladas.

Na tabela 3, foi apresentado o lucro mínimo auferido para os ativos listados com lucro máximo. O destaque para estes mínimos vai para as estratégias numeradas como 11, 12 e 28, sendo que o número de ordens médio não apresentou tanta discrepância em relação aos extremos.

Outros resultados mostram que, para o gráfico de médias móveis, os modelos que merecem destaque são os comportamentos enumerados como 2, 4 e 47. Para as bandas de Bollinger, o modelo de comportamento que se destaca é o 19. Para o histograma de médias, os resultados não se mostraram eficazes.

Também foi realizada a análise dos lucros mínimos para os ativos pesquisados. Na tabela 4, são listados os ativos com as maiores perdas de capital. Alguns pontos devem ser ressaltados, como, por exemplo, a ausência de estratégias baseadas em bandas de Bollinger e de médias móveis. Os dados também mostram que, para a maioria dos ativos destacados abaixo, entra a série original e também a série simulada.

Para os 20 ativos de piores resultados, em 100\% dos casos, a estratégia gráfica que apresentou os subdesempenhos foi o histograma de médias. Outro ponto a ser notado é o de que o lucro máximo, para esses ativos, apresentado na coluna MAX, é muito baixo se comparado a outras estratégias e, em nenhum deles, é auferido lucro, apenas prejuízos líquidos. 
Nesse sentido, duas atitudes poderiam ser tomadas: a primeira é a eliminação da estratégia gráfica histograma de médias e séries simuladas; a segunda é a eliminação dos ativos de menor lucro para compor um sistema autônomo no disparo de ordens.

Quanto aos modelos de comportamento que recebem destaque, cabem aos numerados 1 e 31 os de pior desempenho e, aos que tiveram desempenho melhor, prejuízo menor, os modelos 30, 66, e 70. Nesse caso, a eliminação destes comportamentos de compra deve ser revista ou substituída por outras estratégias, na tentativa de melhorar o desempenho deste tipo gráfico.

\begin{tabular}{llcccccc}
\hline & & & & & & & \\
& & & & & \\
& & MIN & ORDENS & MOD & MAX & № ORDENS & MOD \\
\hline NETC4 & histmed & $\mathbf{1 , 6 9 E - 0 9}$ & 686 & 1 & 0,022126 & 188 & 66 \\
NETC4 & hismedsim & $\mathbf{1 , 7 8 E - 0 9}$ & 684 & 31 & 0,022126 & 188 & 66 \\
VIVO4 & histmed & $\mathbf{6 , 2 E - 0 8}$ & 696 & 1 & 0,251667 & 154 & 69 \\
VIVO4 & hismedsim & $\mathbf{6 , 3 2 E - 0 8}$ & 694 & 31 & 0,251667 & 154 & 69 \\
TCSL3 & histmed & $\mathbf{1 , 0 5 E - 0 7}$ & 746 & 1 & 1,769303 & 194 & 67 \\
TCSL3 & hismedsim & $\mathbf{1 , 0 5 E - 0 7}$ & 746 & 31 & 1,769303 & 194 & 67 \\
RSID3 & histmed & $\mathbf{2 , 0 5 E - 0 7}$ & 500 & 1 & 0,481543 & 136 & 68 \\
RSID3 & hismedsim & $\mathbf{2 , 2 4 E - 0 7}$ & 500 & 31 & 0,481543 & 136 & 68 \\
LIGT3 & histmed & $\mathbf{2 , 8 1 E - 0 7}$ & 726 & 31 & 0,562859 & 158 & 70 \\
LIGT3 & hismedsim & $\mathbf{2 , 8 1 E - 0 7}$ & 726 & 31 & 0,562859 & 158 & 70 \\
TCSL4 & histmed & $\mathbf{4 , 0 4 E - 0 7}$ & 718 & 1 & 1,256897 & 178 & 27 \\
TCSL4 & hismedsim & $\mathbf{4 , 3 6 E - 0 7}$ & 718 & 31 & 1,256897 & 178 & 27 \\
USIM5 & histmed & $\mathbf{5 , 9 6 E - 0 7}$ & 690 & 1 & 6,048427 & 54 & 70 \\
USIM5 & hismedsim & $\mathbf{8 , 1 4 E - 0 7}$ & 682 & 31 & 6,048427 & 54 & 70 \\
ELET3 & histmed & $\mathbf{1 , 3 3 E - 0 6}$ & 720 & 1 & 2,105618 & 172 & 26 \\
ELET3 & hismedsim & $\mathbf{1 , 4 1 E - 0 6}$ & 720 & 31 & 2,119619 & 172 & 26 \\
BRKM5 & histmed & $\mathbf{1 , 9 7 E - 0 6}$ & 676 & 1 & 1,169063 & 144 & 22 \\
BRKM5 & hismedsim & $\mathbf{2 E - 0 6}$ & 674 & 31 & 1,169063 & 144 & 22 \\
TNLP3 & histmed & $\mathbf{2 , 6 3 E - 0 6}$ & 724 & 1 & 1,909503 & 164 & 30 \\
TNLP3 & hismedsim & $\mathbf{2 , 7 5 E - 0 6}$ & 718 & 31 & 1,909503 & 164 & 30 \\
\hline
\end{tabular}

Tabela 4 - Lucro mínimo - gráficos

Outro dado relevante é o número de ordens disparadas para os ativos que tiveram prejuízos extremos. Uma média aproximada se concentra próxima a 687 ordens no horizonte de 10 anos, em média, o que geraria cerca de 69 ordens anuais, número que já pode ser considerado elevado quando se pensa em rendimentos a longo prazo.

Também deve-se levar em conta que, no prejuízo referido acima, não foram descontados os custos de corretagem disparados pelo sistema. Dessa forma, a obtenção do prejuízo máximo se daria em um horizonte menor do que o prazo médio de 10 anos. Para completar a análise das ordens disparadas, são apresentadas as tabelas 5 e 6 de máximos e mínimos de ordens.

Nestas tabelas, dois modelos de comportamentos de compra devem ser enfatizados: o modelo 20, com a totalidade dos mínimos de ordens; e o modelo 1, com a totalidade dos máximos de ordens. O modelo 20 deve ser descartado para futuros testes, pois não produziu nenhuma espécie de negociação no horizonte de 10 anos, aproximadamente. 


\begin{tabular}{cccccccccccc}
\hline Ativo & Graf & MIN & MOD & MAX & MOD & Ativo & Graf & MIN & MOD & MAX & MOD \\
\hline NETC4 & bolling & $\mathbf{0}$ & 20 & 894 & 1 & continuação $\ldots$ & & & & & \\
VIVO4 & bolling & $\mathbf{0}$ & 20 & 988 & 1 & BRTO4 & bolling & $\mathbf{0}$ & 20 & 1030 & 1 \\
BRKM5 & bolling & $\mathbf{0}$ & 20 & 976 & 1 & TNLP4 & bolling & $\mathbf{0}$ & 20 & 1024 & 1 \\
VCPA4+VCPA3 & bolling & $\mathbf{0}$ & 20 & 954 & 1 & TCSL3 & bolling & $\mathbf{0}$ & 20 & 1022 & 1 \\
LIGT3 & bolling & $\mathbf{0}$ & 20 & 1034 & 1 & PCAR5 & bolling & $\mathbf{0}$ & 20 & 1004 & 1 \\
VCPA4+VCPA3 & bolling & $\mathbf{0}$ & 20 & 910 & 1 & GGBR4 & bolling & $\mathbf{0}$ & 20 & 994 & 1 \\
ELET3 & bolling & $\mathbf{0}$ & 20 & 1004 & 1 & TNLP4 & bolling & $\mathbf{0}$ & 20 & 990 & 1 \\
USIM5 & bolling & $\mathbf{0}$ & 20 & 960 & 1 & GFSA3 & bolling & $\mathbf{0}$ & 20 & 350 & 1 \\
TCSL4 & bolling & $\mathbf{0}$ & 20 & 986 & 1 & FHER3 & bolling & $\mathbf{0}$ & 20 & 230 & 1 \\
ELET6 & bolling & $\mathbf{0}$ & 20 & 1022 & 1 & GOAU4 & bolling & $\mathbf{0}$ & 20 & 994 & 1 \\
BRTP4 & bolling & $\mathbf{0}$ & 20 & 1024 & 1 & & & & & & \\
\hline máximo $\ldots$ & & & & & & & & & & & \\
\hline CRUZ3 & bolling & 0 & 20 & $\mathbf{1 1 0 4}$ & 1 & continuação... & & & & & \\
CPLE6 & bolling & 0 & 20 & $\mathbf{1 0 8 0}$ & 1 & SBSP3 & bolling & 0 & 20 & $\mathbf{1 0 4 0}$ & 1 \\
TLPP4 & bolling & 0 & 20 & $\mathbf{1 0 8 0}$ & 1 & VALE3 & bolling & 0 & 20 & $\mathbf{1 0 3 8}$ & 1 \\
TNLP3 & bolling & 0 & 20 & $\mathbf{1 0 6 6}$ & 1 & UGPA4 & bolling & 0 & 20 & $\mathbf{1 0 3 8}$ & 1 \\
AMBEV4 & bolling & 0 & 20 & $\mathbf{1 0 6 2}$ & 1 & TBLE3 & bolling & 0 & 20 & $\mathbf{1 0 3 8}$ & 1 \\
ARCZ6 & bolling & 0 & 20 & $\mathbf{1 0 6 2}$ & 1 & MAGS5+MAGG3 & bolling & 0 & 20 & $\mathbf{1 0 3 6}$ & 1 \\
CGAS5 & bolling & 0 & 20 & $\mathbf{1 0 5 8}$ & 1 & LIGT3 & bolling & 0 & 20 & $\mathbf{1 0 3 4}$ & 1 \\
TRPL4 & bolling & 0 & 20 & $\mathbf{1 0 5 4}$ & 1 & BRTO4 & bolling & 0 & 20 & $\mathbf{1 0 3 0}$ & 1 \\
CMIG4 & bolling & 0 & 20 & $\mathbf{1 0 4 4}$ & 1 & DURA4 & bolling & 0 & 20 & $\mathbf{1 0 3 0}$ & 1 \\
VALE5 & bolling & 0 & 20 & $\mathbf{1 0 4 2}$ & 1 & BRTP4 & bolling & 0 & 20 & $\mathbf{1 0 2 4}$ & 1 \\
LAME4 & bolling & 0 & 20 & $\mathbf{1 0 4 2}$ & 1 & & & & & &
\end{tabular}

Tabela 5 - Número mínimo/máximo de ordens

Na análise dos máximos de ordens, o modelo 1 também deve ser descartado como fonte de eventual utilização em sistemas autônomos, pois, provavelmente, invalidaria o modelo ou o ativo, pelo excesso de custos operacionais, que geraria, em média, cerca de 105 ordens anuais para cada ativo.

Outro ponto que deve ser salientado é o de que em ambos os dados, de mínimos e máximos de ordens, a estratégia gráfica que se evidencia são as bandas de Bollinger, e, neste caso, somente para as séries originais.

Para verificar se as estratégias gráficas foram, de fato, significativas, foi aplicado o teste de bootstrapping, proposto por White (2000), e a estratégia poderia assumir a possibilidade de apresentar retorno anormal nulo, negativo ou positivo, quando comparado ao Ibovespa.

As empresas listadas na tabela 6 foram as que apresentaram as maiores perdas quando comparadas ao Ibovespa. A estatística sugerida fornece um percentual absoluto médio indicativo da diferença quadrada dos retornos obtidos entre as ações e o mercado para cada período em que a estratégia de negociação assumiu a posição comprada.

Nesse sentido, a tabela abaixo (mínimos) sugere que, apesar de menor, o desempenho da estratégia de compra e venda obtida com a utilização do gráfico de médias móveis não apresentou uma magnitude de perda expressiva para a maioria dos ativos analisados. Com relação aos comportamentos de compra/venda que devem ser descartados, são os de numeração 49 e 60, na coluna MOD MIM, pois apareceram com a maior frequência. Na tabela, MOD MAX é o modelo que produziu o resultado da coluna máximo.

Para as ações que obtiveram os melhores desempenhos (máximos), os resultados se mostraram extremamente significativos quanto aos ganhos anormais para as ações da amostra. Estes resultados revelam, de forma semelhante, os mesmos resultados obtidos com o lucro final obtido com os modelos de médias móveis nas tabelas em que foram analisados os lucros das estratégias. Pode-se notar, por consequência, que os valores máximos obtidos, na tabela acima (máximos), com a estatística de White, foram consideravelmente altos. Os comportamentos de melhor desempenho são os de numeração 59 e 70, mais frequentes. 


\begin{tabular}{|c|c|c|c|c|c|c|c|}
\hline & média & mínimo & máximo & desvio & var & $\begin{array}{l}\text { MOD } \\
\text { MÍN }\end{array}$ & $\begin{array}{l}\text { MOD } \\
\text { MAX }\end{array}$ \\
\hline DROG3 & 0,29220 & $-0,34262$ & 9,4102575 & 1,6284301 & 2,6517845 & 60 & 70 \\
\hline ETER3 & $-0,0601534$ & $-0,26262$ & 0,0519364 & 0,095105 & 0,009045 & 49 & 70 \\
\hline MAGS5+MAGG3 & $-0,0250407$ & $-0,24906$ & 0,5522887 & 0,0914401 & 0,0083613 & 49 & 70 \\
\hline UNIP6 & 0,0923606 & $-0,11934$ & 2,5206218 & 0,3255955 & 0,1060124 & 52 & 70 \\
\hline WEGE3 & 0,0356227 & $-0,03024$ & 1,5034701 & 0,2380695 & 0,0566771 & 50 & 70 \\
\hline PRGA3 & $1,228 \mathrm{E}-05$ & $-0,0071$ & 0,0293209 & 0,0045932 & $2,11 \mathrm{E}-05$ & 60 & 70 \\
\hline ENGI4 & $-0,0001344$ & $-0,00495$ & 0,001152 & 0,0008081 & $6,531 \mathrm{E}-07$ & 49 & 68 \\
\hline FFTL4 & 0,0126068 & $-0,003$ & 0,1461897 & 0,024986 & 0,0006243 & 49 & 70 \\
\hline GRND3 & $6,736 \mathrm{E}-05$ & $-0,00293$ & 0,0037796 & 0,0009169 & 8,407E-07 & 66 & 70 \\
\hline EQTL11+EQTL3 & 0,0001367 & $-0,001212$ & 0,0017469 & 0,0004826 & $2,329 \mathrm{E}-07$ & 62 & 70 \\
\hline \multicolumn{8}{|l|}{ máximos ... } \\
\hline AVIL3 & 7084,4955 & 0,000302 & 42551,1 & 11743,146 & 137901468 & 18 & 59 \\
\hline ROMI3 & 0,6608932 & 0,0001196 & 14,32687 & 1,9875463 & 3,9503404 & 12 & 70 \\
\hline RAPT4 & 1,3013665 & 0,000181 & 11,80621 & 2,2761025 & 5,1806425 & 2 & 49 \\
\hline DROG3 & 0,2922 & $-0,342619$ & 9,410258 & 1,6284301 & 2,6517845 & 60 & 70 \\
\hline LREN3 & 0,6114948 & 0,0002658 & 5,612704 & 1,0659961 & 1,1363477 & 9 & 70 \\
\hline CNFB4 & 0,738974 & $8,074 \mathrm{E}-05$ & 5,50469 & 1,1973367 & 1,4336152 & 8 & 70 \\
\hline FESA4 & 0,4720503 & $6,49 \mathrm{E}-05$ & 4,444615 & 0,8175526 & 0,6683922 & 4 & 70 \\
\hline UNIP6 & 0,0923606 & $-0,119344$ & 2,520622 & 0,3255955 & 0,1060124 & 52 & 70 \\
\hline CGRA3 & 0,1357636 & 0,0002036 & 2,264322 & 0,3068091 & 0,0941318 & 5 & 59 \\
\hline FJTA4 & 0,2862381 & 8,493E-05 & 1,859581 & 0,4729873 & 0,223717 & 13 & 69 \\
\hline \multicolumn{8}{|c|}{ médias móveis simuladas ... Mínimos } \\
\hline DROG3 & $-0,6929838$ & $-2,95602$ & $-9,49 \mathrm{E}-05$ & 1,0598255 & 1,1232302 & 49 & 11 \\
\hline LREN3 & 0,1357144 & $-0,42844$ & 0,0006452 & 0,2993632 & 0,0896183 & $69-70$ & 60 \\
\hline ETER3 & $-0,0784498$ & $-0,3434$ & $-4,14 \mathrm{E}-05$ & 0,1204514 & 0,0145086 & 62 & 63 \\
\hline MAGS5+MAGG3 & 0,0517788 & $-0,28605$ & $1,872 \mathrm{E}-05$ & 0,1253524 & 0,0157132 & 51 & 54 \\
\hline WEGE3 & $-0,0398012$ & $-0,18248$ & $-2,56 \mathrm{E}-05$ & 0,0647168 & 0,0041883 & 53 & 11 \\
\hline PRGA3 & 0,0007803 & $-0,00959$ & $1,266 \mathrm{E}-05$ & 0,0042037 & $1,767 \mathrm{E}-05$ & $69-70$ & 57 \\
\hline ABNB3 & $-0,0006504$ & $-0,006$ & $4,421 \mathrm{E}-06$ & 0,0056046 & $3,141 \mathrm{E}-05$ & 59 & 69 \\
\hline SATI3 & 0,0009883 & $-0,00506$ & 0,0001153 & 0,0029323 & 8,598E-06 & 67 & 51 \\
\hline EQTL11+EQTL3 & $-0,0002167$ & $-0,00312$ & $1,839 \mathrm{E}-05$ & 0,0007644 & $5,844 \mathrm{E}-07$ & 50 & 67 \\
\hline ENGI4 & 0,000579 & $-0,00236$ & 0,003873 & 0,00107 & $1,14 \mathrm{E}-06$ & 49 & 55 \\
\hline \multicolumn{8}{|l|}{ máximos ... } \\
\hline AVIL3 & 1675,793 & 0,0003122 & 13726,81 & 3055,291 & 9334803,1 & 41 & 52 \\
\hline LAME4 & 2,6624872 & 0,0002203 & 22,51732 & 4,938316 & 24,386965 & 11 & 69 \\
\hline RAPT4 & 2,4358848 & 0,0002469 & 14,13975 & 3,972708 & 15,782409 & 2 & $69-70$ \\
\hline FJTA4 & 1,34374 & $7,812 \mathrm{E}-05$ & 9,034373 & 2,2049941 & 4,8619988 & 17 & $69-70$ \\
\hline UNIP6 & 0,4907037 & 1,832E-06 & 8,237336 & 1,1780967 & 1,3879118 & 21 & 70 \\
\hline CNFB4 & 0,7293206 & $4,254 \mathrm{E}-05$ & 4,805197 & 1,2412627 & 1,5407331 & 43 & 50 \\
\hline RSID3 & 0,4330592 & 0,0007811 & 3,701393 & 0,8486327 & 0,7201774 & 8 & 58 \\
\hline ROMI3 & 0,5024492 & 4,479E-05 & 3,520876 & 0,8910947 & 0,7940498 & 20 & 49 \\
\hline FESA4 & 0,6343443 & $6,785 \mathrm{E}-05$ & 3,378995 & 1,0180667 & 1,0364599 & 41 & 68 \\
\hline TBLE3 & 0,3128586 & 0,000353 & 2,163097 & 0,5078008 & 0,2578617 & 18 & 54 \\
\hline
\end{tabular}

Tabela 6 - Teste de White - Médias móveis e médias móveis simuladas - mínimos/ máximos

$\mathrm{Na}$ análise das médias móveis simuladas, para as empresas que obtiveram os menores valores da estatística de White (mínimos), nota-se que a grande maioria das empresas que obtiveram desempenhos ruins, nas séries originais, está presente entre as piores nas séries simuladas.

Esses dados sugerem que o sistema de geração de séries simuladas a partir das séries originais produz resultados semelhantes aos dados originais. Com relação aos modelos de comportamento de compra/venda, destacam-se os modelos 49 e 69-70, com maior frequência na obtenção dos piores resultados, devendo ser excluídos de novas pesquisas, optando-se por novos padrões de compra, não testados neste trabalho. Também chama a atenção o modelo 11, frequente entre os de melhor desempenho (MOD MAX), com resultados acima do benchmark para as ações de pior desempenho. 
Os melhores outputs (máximos) obtidos se mostram bastante promissores em relação aos retornos obtidos pelo mercado. Para as primeiras empresas, por exemplo, os resultados mostram diferenças de mais de $1000 \%$ acima do crescimento médio de mercado. Os modelos mais promissores são os de numeração 69 e 70 e, provavelmente, os modelos ao redor destas numerações, tendo em vista que a construção dos modelos foi feita de forma gradual, com pequenas diferenças entre os pares próximos.

Para as Bandas de Bollinger, o procedimento para a validação da análise gráfica foi semelhante. Os piores resultados (mínimos) são apresentados na tabela 7. Nela, verifica-se que os mínimos para o teste de White não foram, em magnitude, tão grandes quanto o esperado, considerando os lucros mínimos obtidos pelo modelo.

Com relação aos modelos do comportamento racional de compra que devem ser excluídos, foram identificados os de numeração 59 e 70, e provavelmente, seus arredores. Outro dado que chama a atenção é o de que os modelos ao redor do de numeração 50 produzem os melhores resultados entre os ativos com pior desempenho.

\begin{tabular}{|c|c|c|c|c|c|c|c|}
\hline & média & mínimo & máximo & desvio & var & MOD MÍN & MOD MAX \\
\hline DROG3 & $-0,2451434$ & $-1,42991$ & $3,771 \mathrm{E}-07$ & 0,5427905 & 0,294625 & $59-70$ & 55 \\
\hline LREN3 & $-0,13646$ & $-0,80333$ & 9,113E-05 & 0,3022151 & 0,091334 & 59 & 49 \\
\hline ETER3 & $-0,0867431$ & $-0,59652$ & $6,901 \mathrm{E}-07$ & 0,1952563 & 0,038125 & 63 & 35 \\
\hline WEGE3 & $-0,0685503$ & $-0,39995$ & 0 & 0,151718 & 0,023018 & 59 & $20-25 ; 44-48$ \\
\hline CGRA3 & $-0,024731$ & $-0,14509$ & 5,648E-06 & 0,0547146 & 0,002993 & 66 & 57 \\
\hline TAMM4 & $-0,0072646$ & $-0,04694$ & 6,571E-05 & 0,020 & 0,000261 & 59 & 50 \\
\hline PRGA3 & $-0,00088835$ & $-0,0100$ & 5,341E-05 & 0,0021556 & $4,64 \mathrm{E}-06$ & 70 & 51 \\
\hline MDIA3 & $-6,269 E-06$ & $-0,00010$ & 0,0001003 & 2,896E-05 & $8,38 \mathrm{E}-10$ & 69 & 60 \\
\hline \multicolumn{8}{|l|}{ máximos.. } \\
\hline AVIL3 & 14462,143 & $-4,7 \mathrm{E}-09$ & 110724,4 & 33083,835 & $1,095 \mathrm{E}+0$ & 25 & 68 \\
\hline RAPT4 & 7,2407474 & $-3,2 \mathrm{E}-07$ & 61,54089 & 16,365914 & 267,8431 & 26 & 66 \\
\hline POMO4 & 0,3324155 & $-5,2 E-06$ & 2,930099 & 0,7683028 & 0,590289 & 36 & 60 \\
\hline LAME4 & 0,2668702 & $-2,3 E-07$ & 1,990811 & 0,6018011 & 0,362164 & 27 & 59 \\
\hline GETI4 & 0,17478 & $-6,0 \mathrm{E}-06$ & 1,758255 & 0,4319959 & 0,186620 & 35 & 70 \\
\hline KLBN4 & 0,1754645 & 0 & 1,680576 & 0,4212375 & 0,177441 & $20-25$ & 68 \\
\hline \multicolumn{8}{|c|}{ bandas de Bollinger simuladas... Mínimos } \\
\hline DROG3 & $-1,1595102$ & $-6,7788$ & $-0,0001679$ & 2,5671449 & 6,590232 & $59-60$ & 48 \\
\hline LREN3 & $-0,1629773$ & $-0,9528$ & 0,0012002 & 0,3618646 & 0,130946 & $59-70$ & 20 \\
\hline ETER3 & $-0,0793992$ & $-0,5045$ & $-1,4 \mathrm{E}-05$ & 0,1758591 & 0,030926 & 59 & 48 \\
\hline UNIP6 & 0,0189009 & $-0,2019$ & 0,4182609 & 0,1290771 & 0,01666 & $67-70$ & $59-62$ \\
\hline TAMM4 & $-0,0066032$ & $-0,0406$ & 0,0004095 & 0,0153846 & 0,00023 & $62-63$ & 48 \\
\hline \multicolumn{8}{|l|}{ máximos.. } \\
\hline AVIL3 & 10378,12103 & 0,00041 & 85487,15 & 23843,012 & 5684892 & 48 & 68 \\
\hline ROMI3 & 2,7315017 & 0,00034 & 15,93209 & 6,0477565 & 36,57535 & $\begin{array}{r}1-19 ; 24-34 \\
39-58\end{array}$ & $59-70$ \\
\hline LAME4 & 1,3366115 & 0,00033 & 7,868266 & 2,9589691 & 8,755498 & 37 & 67 \\
\hline CNFB4 & 1,3177211 & 0,00010 & 7,823703 & 2,9180672 & 8,515116 & 48 & 62 \\
\hline FESA4 & 0,7163647 & 0,00014 & 4,934254 & 1,5915188 & 2,532932 & 27 & 59 \\
\hline RAPT4 & 0,5576038 & $5,5 \mathrm{E}-05$ & 3,889404 & 1,237193 & 1,530646 & 48 & 59 \\
\hline FJTA4 & 0,399929 & $3,7 \mathrm{E}-05$ & 2,332893 & 0,8854982 & 0,784107 & $28 ; 29 ; 43 ; 57 ; 58$ & $60-70$ \\
\hline USIM3 & 0,2549042 & 0,0004 & 1,674857 & 0,5713347 & 0,326423 & 37 & 61 \\
\hline CGRA3 & 0,2731992 & 0,00011 & 1,601296 & 0,6041751 & 0,365027 & 40 & 70 \\
\hline CSNA3 & 0,1700727 & 0,0004 & 0,990027 & 0,3756565 & 0,141117 & $1-58$ & $59-70$ \\
\hline
\end{tabular}

Tabela 7 - Teste de White - Bandas de Bollinger e simuladas - mínimos/máximos 
Já para os melhores resultados (máximos) nas Bandas de Bollinger, os números mostraramse satisfatórios em relação ao produzido pela média de mercado. Com os ativos listados acima, o teste mostrou significância expressiva e positiva.

Em termos reais, esses números também deveriam se traduzir em lucros grandes, caso as estratégias de compra e venda fossem ajustadas, o que, de fato, foi observado nas bandas de Bollinger, em que não foram os maiores lucros, mas foram expressivos. Nesse modelo gráfico, os comportamentos racionais que merecem destaque foram os de numeração 60 e 68 .

Para as bandas de Bollinger simuladas, os resultados foram semelhantes aos das séries originais, sendo que várias das empresas listadas entre as com pior desempenho nas séries originas também estiveram entre as simuladas.

O resultado das cinco empresas com o pior ajuste (mínimos) é, de fato, grande em magnitude, quando comparado ao benchmark de mercado. No entanto, esse resultado é suavizado logo em seguida, com perdas muito próximas do próprio desempenho do lbovespa. Os modelos de comportamento que se destacam são os de numeração 59 a 70.

Quando foram analisados os melhores resultados (máximos) produzidos pelo gráfico de Bollinger, o teste de White sugeriu valores muito superiores aos produzidos pelo Ibovespa. Esse resultado equivale ao encontrado nos lucros produzidos pelas operações de compra/venda nas empresas da amostra. Outro resultado que chama a atenção é a frequência de dois comportamentos racionais entre os melhores resultados, enumerados de 60 e 68, tanto para as séries originais quanto para as séries simuladas.

Para o histograma de médias, a análise de mínimos para o teste de White não aponta perdas tão grandes quanto para os modelos de médias móveis e bandas de Bollinger. A análise também sugere que a queda em termos de magnitude foi suave até se aproximar de 0 ou assumir um retorno próximo ao comportamento do Ibovespa.

Com relação aos modelos de pior ajuste, os que se destacaram foram os de numeração 3, 5, 25, 33 e 42. A ressalva aqui é a de que, mesmo nos modelos que produziram resultados bons para as séries abaixo (coluna MOD MAX), em nenhum deles foi observado algum resultado satisfatório, quando comparados aos resultados das outras estratégias.

Esse problema pode sugerir que os comportamentos racionais não foram adequados ou não atingiram as expectativas esperadas. No entanto, vale lembrar que um número grande de estratégias diferentes foi empregado na tentativa de encontrar algumas que produzissem bons resultados, tentando ainda fugir dos problemas de bootstrapping, mas fazendo-o em essência.

A partir da análise dos máximos, no teste de White, pode-se notar que, para algumas séries, há, de fato, a obtenção de retornos anormais significativos em comparação com o benchmark de mercado. Esse resultado contrasta com a produção de lucros auferida com esse modelo, pois os lucros máximos não chegaram a ser significativos, sendo que na maioria dos casos, apenas ocorreu a manutenção do capital durante os 10 anos negociados.

Com relação aos modelos que produziram os melhores resultados, destaca-se apenas o de numeração 30. Esse modelo, apesar de produzir estatísticas suficientes para mostrar a produção de retornos positivos via teste de White, não foi eficaz na obtenção de lucros.

Para as séries simuladas, considerando o histograma de médias, os piores resultados (mínimos) não se mostraram grandes em termos de magnitude, em relação ao esperado quando se considera os lucros obtidos pelo gráfico. Além disso, a queda dos mínimos é grande e se aproxima do benchmark rapidamente. 


\begin{tabular}{|c|c|c|c|c|c|c|c|}
\hline & média & mínimo & máximo & desvio & var & MOD MÍN & $\begin{array}{l}\text { MOD } \\
\text { MAX }\end{array}$ \\
\hline WEGE3 & $-0,000108$ & $-0,00012$ & $-9,42 \mathrm{E}-05$ & $5,41 \mathrm{E}-06$ & $2,935 \mathrm{E}-11$ & $2 ; 3 ; 5 ; 7 ; 32 ; 33$ & 70 \\
\hline TAMM4 & $-2,41 \mathrm{E}-05$ & $-1 \mathrm{E}-04$ & $4,605 \mathrm{E}-05$ & $4,73 \mathrm{E}-05$ & $2,241 \mathrm{E}-09$ & 25 & 13 \\
\hline ETER3 & $-7,43 \mathrm{E}-05$ & $-9 \mathrm{E}-05$ & $-6,06 \mathrm{E}-05$ & $6,12 \mathrm{E}-06$ & $3,75 \mathrm{E}-11$ & 3 & 30 \\
\hline ABNB3 & $-5,87 \mathrm{E}-05$ & $-8,9 E-05$ & 4,219E-05 & $4,21 \mathrm{E}-05$ & $1,774 \mathrm{E}-09$ & 42 & 38 \\
\hline PRGA3 & $-3,71 \mathrm{E}-05$ & $-8,2 \mathrm{E}-05$ & $1,999 \mathrm{E}-05$ & $3,67 \mathrm{E}-05$ & $1,35 \mathrm{E}-09$ & 33 & 69 \\
\hline CGRA3 & $3,59 \mathrm{E}-06$ & $-6,1 \mathrm{E}-05$ & $4,142 \mathrm{E}-05$ & $2,34 \mathrm{E}-05$ & $5,496 \mathrm{E}-10$ & 51 & 42 \\
\hline DROG3 & $-3,09 E-05$ & $-4,4 \mathrm{E}-05$ & $7,797 \mathrm{E}-07$ & $1,17 \mathrm{E}-05$ & $1,387 \mathrm{E}-10$ & 29 & $30 ; 68-70$ \\
\hline CPFE3 & $-1,72 \mathrm{E}-05$ & $-3,7 \mathrm{E}-05$ & $2,049 \mathrm{E}-06$ & $8,84 \mathrm{E}-06$ & $7,816 \mathrm{E}-11$ & 5 & 19 \\
\hline MAGS5+MAGG3 & $-1,32 \mathrm{E}-05$ & $-2,9 \mathrm{E}-05$ & $-2,89 \mathrm{E}-06$ & $6,29 \mathrm{E}-06$ & $3,965 \mathrm{E}-11$ & 33 & 19 \\
\hline ODPV3 & $-1,52 \mathrm{E}-05$ & $-2,68 \mathrm{E}-05$ & $5,49 \mathrm{E}-06$ & $7,47 \mathrm{E}-06$ & $5,592 \mathrm{E}-11$ & $24-64$ & 34 \\
\hline \multicolumn{8}{|l|}{ máximos... } \\
\hline NETC4 & 0,0009549 & 0,0008345 & 0,001044 & 5,53E-05 & 3,059E-09 & 31 & 64 \\
\hline RSID3 & 0,0008973 & 0,0008177 & 0,000967 & $3,77 \mathrm{E}-05$ & $1,428 \mathrm{E}-09$ & 32 & $12 ; 44 ; 52$ \\
\hline MMXM3 & 0,0006562 & 0,0004859 & 0,000773 & $7,92 \mathrm{E}-05$ & $6,275 \mathrm{E}-09$ & 38 & $16 ; 48 ; 56$ \\
\hline AVIL3 & 0,0005687 & 0,0004791 & 0,000708 & $6,37 \mathrm{E}-05$ & 4,06E-09 & 59 & 5 \\
\hline VIVO4 & 0,0004911 & 0,0004392 & 0,000538 & 1,87E-05 & $3,496 \mathrm{E}-10$ & 1 & 63 \\
\hline TEND3 & 0,0004652 & 0,000406 & 0,000514 & $2,48 \mathrm{E}-05$ & $6,154 \mathrm{E}-10$ & 1 & 30 \\
\hline GFSA3 & 0,0004412 & 0,0003364 & 0,000488 & 4,53E-05 & $2,055 \mathrm{E}-09$ & 34 & $45 ; 53$ \\
\hline BBRK3 & 0,0004299 & 0,0004053 & 0,000486 & $1,68 \mathrm{E}-05$ & $2,825 \mathrm{E}-10$ & 60 & 38 \\
\hline LIGT3 & 0,0004085 & 0,0003542 & 0,000471 & $3,44 \mathrm{E}-05$ & $1,187 \mathrm{E}-09$ & 5 & 30 \\
\hline CYRE3 & 0,0004085 & 0,0003255 & 0,00046 & $3,96 \mathrm{E}-05$ & $1,575 \mathrm{E}-09$ & 41 & 21 \\
\hline \multicolumn{8}{|c|}{ histograma de médias simuladas ... Mínimos } \\
\hline WEGE3 & $-0,000108$ & $-0,00012$ & $-6,14 \mathrm{E}-05$ & $7,82 \mathrm{E}-06$ & $6,115 \mathrm{E}-11$ & $2 ; 3 ; 5 ; 7 ; 32 ; 33$ & 1 \\
\hline TAMM4 & $-2,40 \mathrm{E}-05$ & $-1 \mathrm{E}-04$ & $4,605 \mathrm{E}-05$ & $4,72 \mathrm{E}-05$ & $2,228 \mathrm{E}-09$ & 25 & 13 \\
\hline ETER3 & $-7,44 \mathrm{E}-05$ & $-9 \mathrm{E}-05$ & $-6,06 \mathrm{E}-05$ & $6,32 \mathrm{E}-06$ & $4,005 \mathrm{E}-11$ & 3 & 30 \\
\hline ABNB3 & $-5,81 \mathrm{E}-05$ & $-8,9 \mathrm{E}-05$ & $7,675 \mathrm{E}-05$ & $4,35 \mathrm{E}-05$ & 1,9E-09 & 42 & 1 \\
\hline PRGA3 & $-3,66 \mathrm{E}-05$ & $-8,2 \mathrm{E}-05$ & 1,999E-05 & $3,66 \mathrm{E}-05$ & $1,34 \mathrm{E}-09$ & 33 & 69 \\
\hline CGRA3 & $7,408 \mathrm{E}-06$ & $-6,1 \mathrm{E}-05$ & 0,0002335 & $3,58 \mathrm{E}-05$ & $1,282 \mathrm{E}-09$ & 51 & 1 \\
\hline DROG3 & $-3,05 E-05$ & $-4,4 \mathrm{E}-05$ & $7,797 \mathrm{E}-07$ & $1,19 \mathrm{E}-05$ & $1,425 \mathrm{E}-10$ & 29 & $30 ; 68-70$ \\
\hline CPFE3 & $-1,69 \mathrm{E}-05$ & $-3,7 \mathrm{E}-05$ & $3,794 \mathrm{E}-06$ & $9,18 \mathrm{E}-06$ & 8,4310659 & 5 & 1 \\
\hline MAGS5+MAGG3 & $-1,29 E-05$ & $-2,9 E-05$ & $-2,89 \mathrm{E}-06$ & $6,22 \mathrm{E}-06$ & $3,877 \mathrm{E}-11$ & 33 & 19 \\
\hline ODPV3 & $-1,52 \mathrm{E}-05$ & $-2,68 \mathrm{E}-05$ & $5,49 \mathrm{E}-06$ & $7,45 \mathrm{E}-06$ & $5,558 \mathrm{E}-11$ & $24-64$ & 34 \\
\hline \multicolumn{8}{|l|}{ máximos... } \\
\hline NETC4 & 0,000959 & 0,0008345 & 0,00112 & $5,68 \mathrm{E}-05$ & $3,226 \mathrm{E}-09$ & 31 & 1 \\
\hline RSID3 & 0,0009014 & 0,0008177 & 0,001107 & $4,42 \mathrm{E}-05$ & 1,954E-09 & 31 & 1 \\
\hline AVIL3 & 0,0005727 & 0,0004791 & 0,000918 & $7,58 \mathrm{E}-05$ & $5,75 \mathrm{E}-09$ & 59 & 1 \\
\hline MMXM3 & 0,0006584 & 0,0004859 & 0,000773 & $7,74 \mathrm{E}-05$ & 5,993E-09 & 38 & $16 ; 48 ; 56$ \\
\hline USIM3 & 0,0003898 & 0,0003303 & 0,000608 & $4,03 \mathrm{E}-05$ & $1,627 \mathrm{E}-09$ & 37 & 1 \\
\hline VIVO4 & 0,0004931 & 0,0004446 & 0,000581 & $2,05 \mathrm{E}-05$ & $4,239 \mathrm{E}-10$ & 31 & 1 \\
\hline TCSL3 & 0,000386 & 0,0003527 & 0,000573 & $3,06 \mathrm{E}-05$ & $9,369 \mathrm{E}-10$ & 38 & 1 \\
\hline GFSA3 & 0,000444 & 0,0003364 & 0,000559 & 4,64E-05 & 2,159E-09 & 34 & 1 \\
\hline TEND3 & 0,0004673 & 0,0004062 & 0,000553 & $2,59 \mathrm{E}-05$ & $6,72 \mathrm{E}-10$ & 31 & 1 \\
\hline CYRE3 & 0,0004114 & 0,0003255 & 0,000552 & 4,26E-05 & 1,817E-09 & 41 & 1 \\
\hline
\end{tabular}

Tabela 8 - Teste de White - Histograma de médias e simuladas - mínimos/máximos

O ponto interessante, nessa tabela, é o de que o modelo 33 aparece com extrema frequência, seguido dos modelos 3, 5, 25 e 32. Coincidentemente, estes modelos aparecem entre os de pior eficiência entre os de pior ajuste para as séries originais, sugerindo a eliminação definitiva desses comportamentos como estratégias de compra/venda. 
Em relação aos resultados máximos do teste de White, os números se mostraram bastante significativos em termos de magnitude. Considerando esses valores, esperava-se que as estratégias de compra/venda produzissem grandes lucros, o que, na prática, não foi evidenciado. Tal como aconteceu para as séries originais, esses resultados poderiam sugerir comportamentos de compra/ venda mal formulados ou a presença de aleatoriedade nas estratégias criadas, com o intuito de produzir lucros, e, junto com isso, problemas de bootstrapping.

Quanto aos modelos que se destacaram, um comportamento que aparece com grande frequência entre os melhores é o de numeração 1. Também chama atenção o modelo 31, responsável por produzir os piores resultados entre os ativos de melhores desempenhos.

Na tentativa de aumentar o empirismo dos resultados gráficos, foi aplicado o teste proposto por Lorenzoni et al. (2007), com as modificações estruturais propostas pelo autor na obtenção da fabricação das séries simuladas. O teste foi aplicado não só para as séries simuladas como também para as séries originais dos retornos dos ativos.

Nesse sentido, foram realizadas comparações não paramétricas através do teste quiquadrado, para a validação de diferenças de médias. A aplicação das estratégias gráficas indicou posição comprada para os retornos das ações versus os retornos do Ibovespa, no período idêntico à posição comprada. Os testes foram feitos para todos os tipos de análise gráfica e aplicados em todo o período, conjuntamente.

A estatística mostrada na tabela 9 traz a seguinte informação: se qui-quadrado for igual a zero (0), então há diferença significativa entre os retornos das séries, quando comparadas ao Ibovespa, no mesmo período. Os outros números internos, 20-25, por exemplo, para as bandas de Bollinger com relação ao ativo de código ALLL11, mostram os modelos de comportamento racional em que não foram evidenciadas diferenças significativas no período analisado.

Para a estatística modificada de Lorenzoni et al. (2007), proposta pelo autor, alguns resultados merecem destaque: para os gráficos de médias móveis e para o histograma de médias (séries originais e simuladas), em nenhuma das séries e para nenhum dos comportamentos racionais adotados foi encontrada igualdade de médias, ou seja, todos os resultados sugeriram diferença de médias significativa para todos os resultados. Conforme as constatações anteriores, a estatística sugere que as diferenças sejam significativas e positivas para a utilização de médias móveis e significativas e negativas para a utilização de histogramas de médias e suas simulações.

Para os ativos SMTO3 e CYRE3, em nenhuma das séries foram encontradas diferenças estatísticas positivas ou negativas e para nenhum comportamento racional de compra adotado. Isso leva a duas suposições: a primeira, de que podem ter ocorrido poucos negócios para esses ativos; e a segunda, de que houve muitos negócios no período e, por casualidade, as séries possuíram crescimento ou perdas semelhantes ao Ibovespa quando a estratégia de bandas de Bollinger simuladas assumiu posição comprada/vendida. 


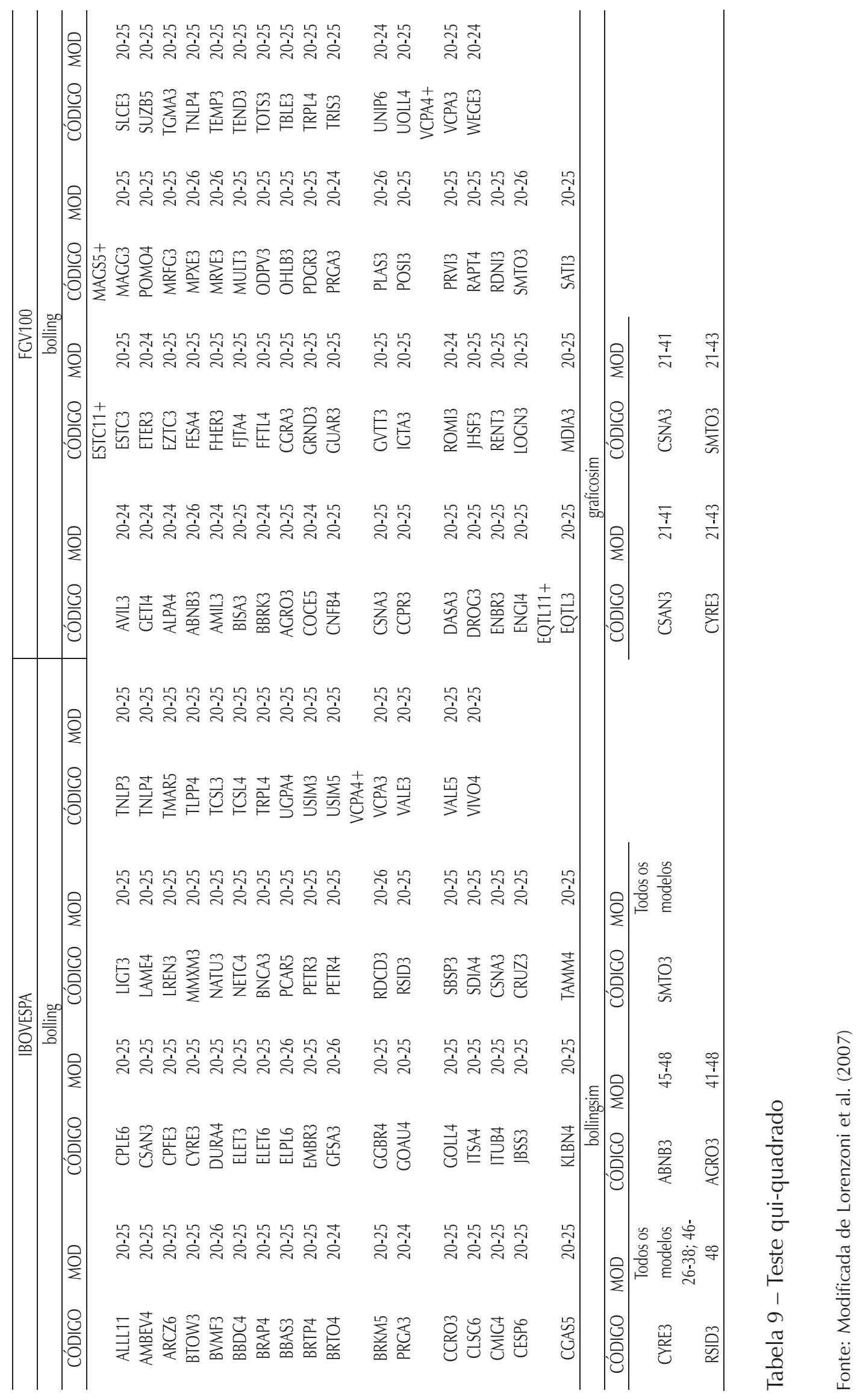


Já para as empresas RSID3, ABNB3 e AGRO3, alguns dos comportamentos enumerados entre 41 e 48 não apresentaram resultados positivos ou negativos para as diferenças de médias. Assim, pode-se considerar que essas séries possuem peculiaridades que não produziram resultados satisfatórios para esses comportamentos, com o gráfico de bandas de Bollinger simuladas. Nesse caso, na maior parte dos ativos negociados, os comportamentos racionais de compra enumerados de 20 a 26 produziram resultados semelhantes ao Ibovespa, ou seja, a diferença de média não foi constatada. A ressalva aqui é a de que não poderia haver, de fato, diferença de médias para esses modelos, porque não houve disparos de ordens de compra ou venda para todo o período analisado. Nesse sentido, esse tipo de comportamento deve ser retirado, e novos tipos de comportamentos devem ser incorporados.

Para os ativos CSAN3, CYRE3 e SMTO3 e para os comportamentos racionais enumerados de 21 a 43, eles também não produziram resultados satisfatórios, quando comparados ao Ibovespa. Vale ressaltar que, nesse caso, o problema, talvez, não seja a formação dos comportamentos racionais, e sim a própria característica intrínseca das séries apontadas.

\section{CONCLUSÃO}

Este trabalho procurou evidências de performance comparativa, da possibilidade de automação empírica e do desenvolvimento de comportamentos racionais de compra/venda modificados para alguns dos gráficos utilizados pelos traders ou operadores de mesa de operação/ homebroker para os ativos financeiros da Bovespa.

Para isso, foi executada uma bateria de cálculos estatísticos de validação das estratégias conjuntas para cada categoria de análise gráfica. Os comportamentos racionais de compra/ venda possuíam a finalidade única de maximizar a riqueza do investidor no período amostral e não a função ou a utilidade.

Tentou-se replicar a estratégia gráfica utilizada no dia a dia dos traders. Nesse sentido, não foram feitas previsões, e sim houve uma tentativa de testar vários comportamentos que, supostamente, seriam adequados para cada tipo de gráfico testado. O comportamento racional foi utilizado com o auxílio dos sinais de compra/venda emitidos pelos gráficos utilizados neste estudo para o disparo de ordens. Sendo assim, a compra só foi realizada quando o gráfico produziu um comportamento indicativo de compra, e a venda só foi realizada quando o gráfico produziu a condição ideal de venda.

As séries históricas utilizadas para comporem a amostra possuíam memória longa de dados, com data inicial de corte o dia 01/01/2000 e data final o dia 30/09/2009, perfazendo um total de 10 anos, aproximadamente. Para os ativos que não possuíram a totalidade de dias de negociação, foram utilizados os períodos disponíveis na base de dados.

A utilização de médias móveis com alguns dos comportamentos testados produziu resultados muito superiores ao alcançado pelo Ibovespa, seguindo alguns dos resultados encontrados em Sullivan, Timmermann e White (1999) e Lo, Mamaysky e Wang (2000).

O gráfico que obteve os melhores resultados foi o de médias móveis, seguido pelas bandas de Bollinger, com a produção de resultados inferiores aos de médias móveis, mas superiores aos produzidos pelo benchmark utilizado. Já para o histograma de médias, os resultados não se mostraram eficientes na grande maioria dos casos.

Outro dado que chamou a atenção foi o de que a criação de séries simuladas a partir das séries originais e a aplicação de comportamentos racionais sobre elas também mostraram resultados significativos, exibindo a presença de conteúdo informacional. Isso resulta em um 
princípio para uma abordagem de cunho estatístico que possa validar fortemente a abordagem gráfica, com a criação de séries simuladas, extraindo-se das originais algumas características como média e desvio padrão.

Para trabalhos futuros, recomenda-se a continuação desta metodologia de trabalho, com uma maior exploração de comportamentos racionais modificados de compra/venda de ativos e a utilização de outros modelos para comparação com os de melhores resultados obtidos neste trabalho. Recomenda-se também a introdução da lógica interanálises, ou seja, a estratégia gráfica só poderia ser utilizada nos casos em que uma análise fundamentalista prévia indicasse sucesso ou fracasso para a empresa.

Nesse sentido, este trabalho cumpriu com seus objetivos, oferecendo evidências empíricas da possibilidade da robotização de operações em bolsas ou de séries de qualquer outra espécie, seja para compra de ações, dólares, commodities etc., seja para disparos de ordens e análises de cenários independentes de opiniões subjetivas através da análise gráfica.

\section{REFERÊNCIAS BIBLIOGRÁFICAS}

ARROW, K. J. Rational choice functions and orderings. Economica, v. 26, p. 12-127, 1959.

BAJGROWICZ, P.; SCAILLET, O. Technical trading revisited: persistence tests, transaction costs, and false discoveries. Swiss Finance Institute Research Paper n. 05-08. Available at SSRN: < http://ssrn.com/ abstract $=1095202>$. Acesso em: 1 Jan. 2008.

BATCHELOR R. A.; KWAN, T. Y. Judgmental bootstrapping of technical traders in the bond market. International Journal of Forecasting, v. 23. Special Issue on Judgmental Forecasting, n. 3. p. 427-445, 2007.

BROCK, W.; LAKONISHOK, J.; LEBARON, B. Simple Technical Trading Rules and the Stochastic Properties of Stock Returns. Journal of Finance, v. 47, n. 5, p. 1731-1764, 1992.

COWLES, A. Can stock market forecasters forecast? Econometrica, v. 1, p. 309-324, 1933.

EDWARDS, R. D.; MAGEE, J. Technical analysis of stock trends. $5^{\text {th }}$ ed. Springfield: John Magee, 1966.

FAMA, E. F. Efficient capital markets: a review of theory and empirical work. Journal of Finance, $v$. 25, p. 383-417, 1970.
FOCK, J. H.; KLEIN, C.; ZWERGEL, B. Performance of candlestick analysis on intraday futures data. The Journal of Derivatives, v. 13, n. 1, p. 28-40, Fall, 2005.

GARTLEY, H. M. Profits in the stock market. Pomeroy, Washington: Lambert-Gann Publishing Company, 1935.

JEGADEESH, N. Discussion. Journal of Finance, v. 55, p. 1765-70, 2000.

LORENZONI et al. On the statistical validation of technical analysis. Revista Brasileira de Finanças, v. 5, p. 3-28, 2007.

LO, A.; MAMAYSKY, H.; WANG, J. Foundations of technical analysis: Computational algorithms, statistical inference, and empirical implementation. Journal of Finance, v. 55, n. 4, p. 1705-1765, 2000.

MARKOWITZ, H. M. Portfolio Selection. Journal of Finance, v. 7, n. 1, p. 77-91, 1952.

MURPHY, John J. Technical analysis of the futures markets. Englewood Cliffs, N. J.: New York Institute of Finance, 1986.

NADARAYA, E. A. On estimating regression. Theory of Probability and its Applications, v. 10, p. 186190, 1964. 
PIETRO NETO, J. Uma comparação empírica do desempenho preditivo das análises fundamentalista, gráfica e econométrica no mercado acionário brasileiro. Tese (Doutorado em Administração - Finanças)-Universidade de Brasília, Brasília, 2011.

ROSS, S. A. The arbitrage theory of capital asset pricing. Journal of Economic Theory, v. 13, p.341360, 1976.

SAFFI, P. A. C. Análise técnica: sorte ou realidade? Revista Brasileira de Economia, v. 57, n. 4, p. 953-974, 2003.

SAMUELSON, P. A. Proof that properly anticipated prices fluctuate randomly. Industrial Management Review, v. 6, p. 41-49, 1965.

SHARPE, W. F. Capital asset prices: a theory of market equilibrium under conditions of risk. Journal of Finance, v. 19, n. 3, p. 425-442, 1964.
SULLIVAN, R.; TIMMERMANN, A.; WHITE, H. Data-snooping, technical trading rules and the bootstrap. Journal of Finance, v. 54, p. 1647-1692 1999.

TREYNOR, J. L.; FERGUSON, R. In defense of technical analysis. Journal of Finance, v. 40, p. 757 772, 1985.

WATSON, G. S. Smooth regression analysis. Sankya A, v. 26, p. 359-372, 1964.

WANG, J. L.; CHAN, S. H. Stock market trading rule discovery using pattern recognition and technical analysis Source Expert Systems with Applications. International Journal archive, v. 33, issue 2, p. 304-315, 2007.

WHITE, H. A reality check for data snooping. Econometrica, v. 68, p. 1097-1126, 2000. 